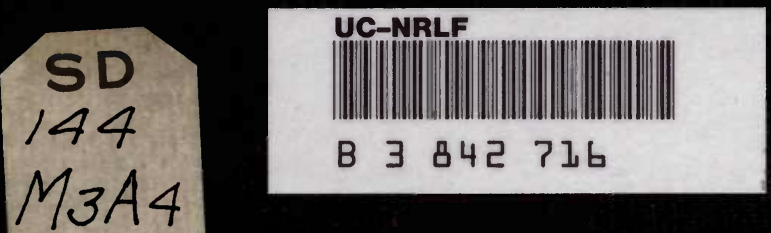




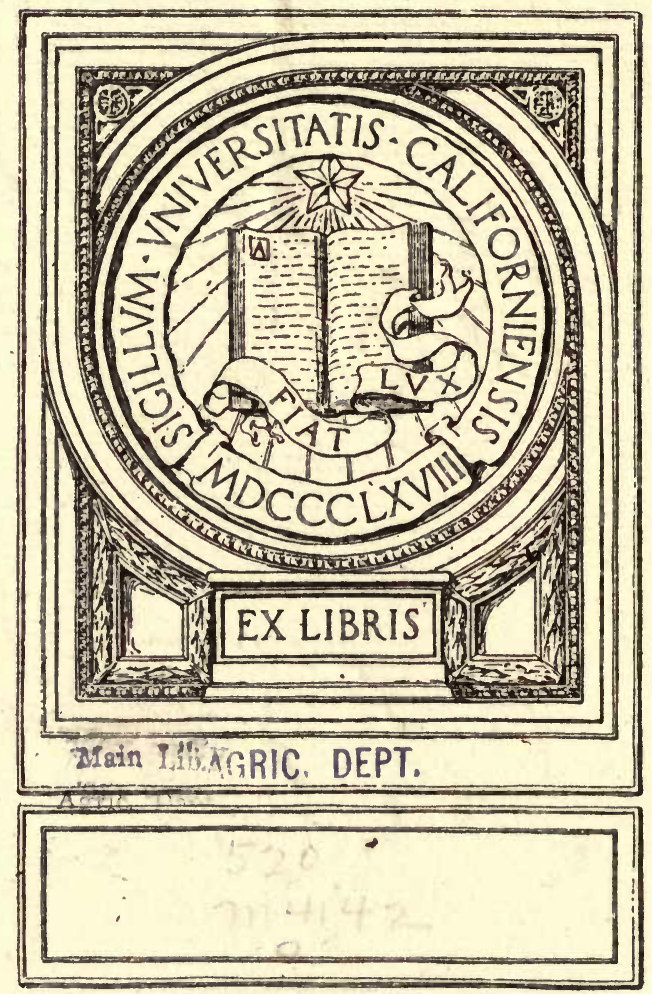








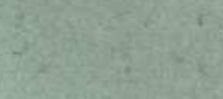

â.

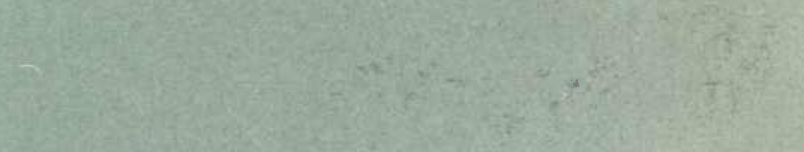




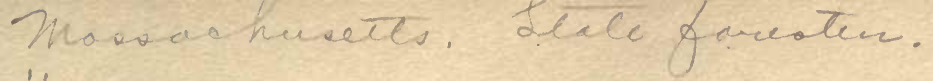

\section{THE OLDER}

\section{Forest Plantations}

IN

\section{MASSACHUSETTS.}

\section{CONIFERS.}

J. R. Simmons, Assistant Forester, under the Direction of F. W. Rane, State Forester.

BOSTON:

WRIGHT \& POTTER PRINTING CO., STATE PRINTERS, 32 DERNE STREET. 
APPROVED BY

The State Board of Publication.

AGRIC. DEPT.

$2 \pi 2: 17 i b 0$

$m^{3}$

$x^{x}$

5

$n^{3}$

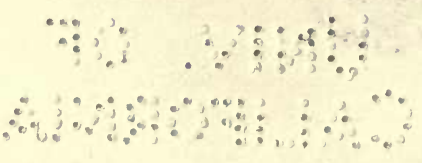




\section{FOREW ORD.}

Mr. Simmons has brought together in this bulletin some very valuable information. All of the examples cited are results of actual early planting in this State.

Many of the plantations, it may be said, have not had the normal conditions that would exist to-day. Most of the stock planted was wild stock dug from the surrounding country, and in some instances the land used was extremely inferior.

With nursery-grown transplants, adaptable soils and modern methods of thinning far better results may be had in the future.

The results reported herewith are extremely conservative, and the reader, I am sure, cannot help receiving encouragement in attempting forest planting of pine in this State.

Interest in reforestation is growing rapidly each year, and we may anticipate what our well-directed efforts of to-day. will bring forth twenty-five to fifty years hence by the results here shown.

Every 1,000 acres planted now will mean much to posterity, and, as well, reflect great credit and profit to our generation.

$$
\text { F. W. RANE, }
$$

Feb. 1, 1915.

State Forester. 



\section{FOREST PLANTATIONS IN MASSACHUSETTS.}

\section{INTRODUCTION.}

The object of this bulletin is to show the practicability and results of forest planting of coniferous trees, and deals with plantations which have reached an age when value can be measured in terms of lumber. The tracts selected for observation were originally planted by private individuals who represent the pioneers in the work of reforestation in this State. By interviewing these owners, or those to whom their property has been handed on, a considerable amount of information has been collected with regard to the objects which they had in mind in the beginning, the methods employed and the later management of their plantations. To this has been added measurements of each wood lot, either as a whole, or by the selection of sample plots, and the contents computed as described in the succeeding pages. The data and accompanying illustrations were taken in 1914. It is the desire of the State Forester to encourage those who own waste land to bring it back into forest production. This investigation represents one method of showing what may be expected from such an undertaking.

\section{Early Methods and Interest in Forest Planting.}

The period between 1820 and 1880 was one of enthusiastic planting of pine in New England. The lumberman foresaw the time when natural white pine as a marketable commodity would be gone, and the rise in prices would make planted timber of economic importance. Large plantations were made by private owners, and a few by corporations. Seedlings were usually dug up from the fields lying around old seed pines, and planted either at random or in rows, and spaced at distances 
varying from 4 to 15 feet. Seed plots were attempted by some, and others even tried out broadcast sowing. At the end of this period there were in Massachusetts alone forest plantations of white pine to the extent of 10,000 acres. $^{1}$

Typical of the forest planters of this time was Mr. Augustus Pratt, a former member of the State Board of Agriculture, who, when nineteen years old, planted pine seed on an old pasture belonging to his father. The wood lot which thus developed has been recently cut, and was between forty and fifty years old.

After 1880 interest began to decline, chiefly because of the immense supply of lumber brought from the region of the Great Lakes at a low rate of transportation and the inadequate methods of combating forest fires; these conditions tended to gradually dampen the enthusiasm of the forest planter.

\section{Present Need of Reforestation.}

We are now entering once more upon a campaign for the reclamation of waste land. This is due not alone to the decrease in our supply of lumber, but also to the following facts:-

There is a growing sentiment among our people for forests and scenic beauty. There are nearly 1,000,000 acres of waste land in Massachusetts; our hardwood forests are threatened by gypsy, brown-tail and other moth pests, and our beautiful chestnut tracts by the ever-increasing chestnut bark disease. Improved methods of forest-fire fighting and the co-operation of railroads, local fire departments and individuals have made possible the protection of forests, once they have been acquired.

Coniferous trees offer the best means of realizing our present needs. They act as the most effective check upon the devastations of the moth, being unedible to the brown-tail, while the gypsy will pass them by if he can find anything else upon which to subsist. ${ }^{2}$ Beneath a pine forest there is always a thick bed of needles which keeps the ground moist and free from sprouts and deciduous seedlings. Therefore, while pine develops tremendous heat in case of fire, it prevents the collec-

1 United States Forest Service Bulletin No. 35.

2 See Massachusetts State Forester's Bulletin on "Improvement Thinnings." 
tion of inflammable material which would feed on ordinary ground fire; in other words, it is a good preventive against the first causes.

\section{Recent Plantations.}

Excellent plantations of pine, ranging from trees a few inches in height to 15 or 20 feet, may be seen on the watersheds of many of our lakes and streams. A large number of individuals and corporations in all parts of the State have undertaken reforestation, either upon their own initiative or with the cooperation of the State Forester. In 1914 the amount of land planted under the reforestation act was 550 acres. In addition to this, the Massachusetts State institutions and commissions planted about 700 acres. The transplants were supplied from the State Forester's nursery at Amherst, Mass. A new State Forest Commission has, within the year, been appointed by the Governor, and empowered to acquire wild and waste lands for the purpose of converting them into State forests. These lands will be turned over to the State Forester for planting and management. In most parts of the State the work of private forestry companies is also becoming evident. To many people a flourishing forest is sufficient remuneration. For the economic advantages of reforestation the reader is referred to the measurements given herewith, and also to "Forest Mensuration of the White Pine," which can be obtained by writing to the State Forester.

\section{The Forest Taxation Law.}

The advantages of the new forest taxation law should be known and understood by those interested in the subject of reforestation. In order to benefit by the provisions of the law the forest plantation must first be registered. The law then substitutes for the general tax on land and timber two taxes, i.e., one on the land at its own value, the same as if all the trees had been removed, and one equal to 6 per cent. of the stumpage value, payable when the timber is cut. The owner of a registered plantation would therefore pay an annual tax on the value of his land ( $\$ 1$ to $\$ 10$ per acre, which at a $\$ 20$ rate would amount to 2 to 20 cents) so long as the plantation stood, and a yield tax of 6 per cent. when the timber was cut 
$\$ 10$ to $\$ 20$ per acre): "These two taxes would be considerably less than the amount paid under the present system, and their amount would be a known quantity, because the owner could determine in advance the amount which he would have to expend. Under the old system he would be subject to the judgment of his assessors, who can raise the amount of his valuation from year to year. The reader is referred to the Massachusetts State Forester's Bulletin, "The Forest Taxation Law."

\section{Plantations.}

South Orleans, Mass. - Owner, John Kenrick.

History. - This plantation was made in March, 1876, by John Kenrick, Sr., and consists of about 7 acres of white pine, Scotch pine and American larch. Previous to planting, the land had borne rye for one year and corn for two years, and the trees were set out on the corn stubble 4 feet apart. On a portion of the tract, instead of using seedlings, small seed spots were sown, always with the same spacing. The larch consisted entirely of seedlings. In spite of the close spacing, the trees have made a remarkable growth for this section of the State, and there are very few blank spaces, even where the seed was used. Occasionally a small group of trees more slender than the others marks where several seedlings sprang up as if from a single root out of the seed spot, but usually the most vigorous tree has crowded out the others.

Objects. - Personal interest in forest planting, and to demonstrate the practicability of forest planting on Cape Cod; to improve and utilize land otherwise of little agricultural value.

Treatment. - The original owner lived to make his first forest thinning, though well on in years when the planting was done. This was confined almost entirely to removing the dead and overcrowded trees. The 4-foot spacing remains in evidence throughout most of the tract. A large number of trees could now be removed to advantage, but even as they stand they present a marked contrast to the native pitch pine, being taller and of greater diameter. 


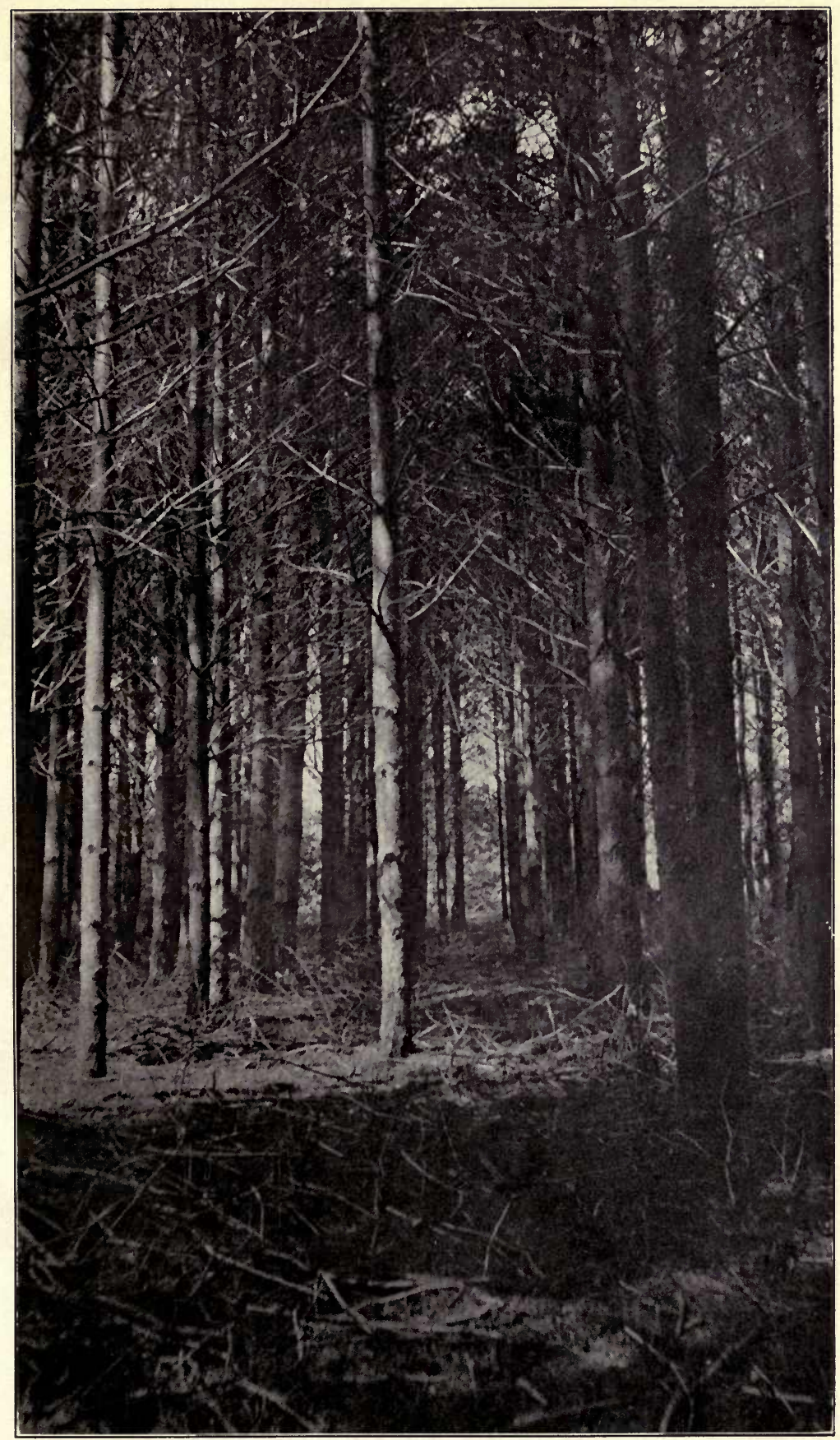

Thirty-eight year old plantation of white pine. John Kenrick, South Orleans, Mass. 



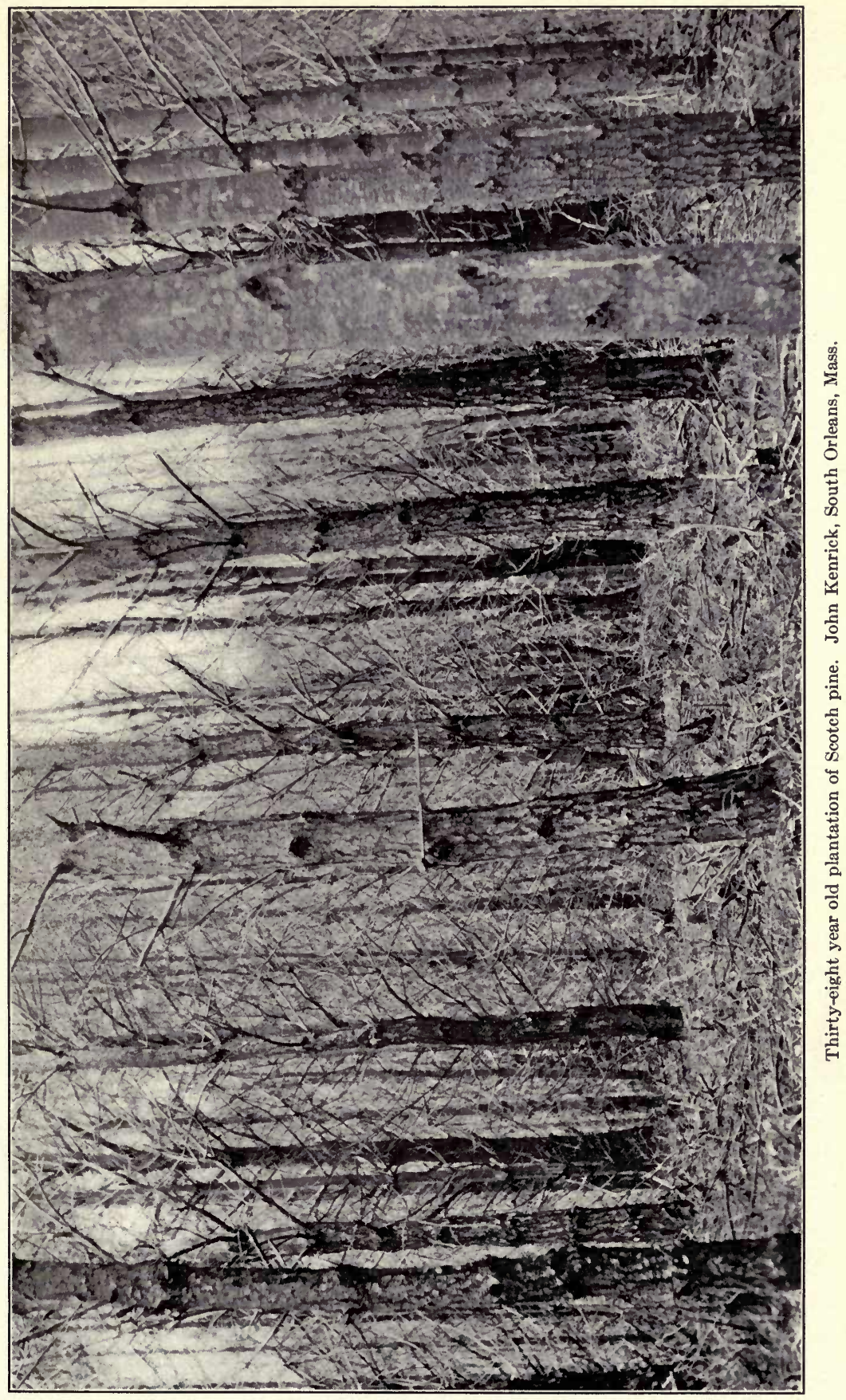



Plot No. 1. White Pine predominating.

Sample plot, 280 by 110 feet; age, thirty-eight years.

\begin{tabular}{|c|c|c|c|c|c|c|c|c|c|c|}
\hline & \multirow{2}{*}{\multicolumn{2}{|c|}{ Diameter }} & & & \multirow[b]{2}{*}{ (INCHES). } & & \multicolumn{3}{|c|}{ NUMBER OF TREES. } & \multirow{2}{*}{$\begin{array}{l}\text { Board } \\
\text { Feet. }\end{array}$} \\
\hline & & & & & & & $\begin{array}{l}\text { White } \\
\text { Pine. }\end{array}$ & $\begin{array}{l}\text { Scotch } \\
\text { Pine. }\end{array}$ & $\begin{array}{l}\text { Height } \\
\text { (Feet). }\end{array}$ & \\
\hline 5, & . & - & . & - & . & & 70 & 25 & 35 & 1,188 \\
\hline 6 & . & - & . & - & . & - & 59 & 21 & 35 & 1,400 \\
\hline 7 , & . & - & . & - & . & . & 55 & 23 & 35 & 1,950 \\
\hline 8, & . & - & . & . & . & - & 38 & 21 & 40 & 2,065 \\
\hline 9, & & - & . & . & . & - & 21 & 14 & 40 & 1,575 \\
\hline 10, & . & $\cdot$ & . & . & . & - & 16 & 8 & 40 & 1,320 \\
\hline 11, & . & - & - & - & . & - & 8 & 6 & 45 & 1,085 \\
\hline 12, & . & . & . & - & . & - & 7 & 2 & 45 & 810 \\
\hline 13, & . & . & . & - & . & - & - & 4 & 45 & 410 \\
\hline 14, & & . & . & . & . & - & 1 & - & 45 & 120 \\
\hline & Total, & . & . & . & . & . & 275 & 125 & $40^{1}$ & 11,923 \\
\hline
\end{tabular}

Total number of trees to acre, 571.

Total board feet to acre, 17,033 .

1 Average height.

Plot No. 2. Scotch Pine predominating.

Area, $1 / 4$ acre; age, thirty-eight years.

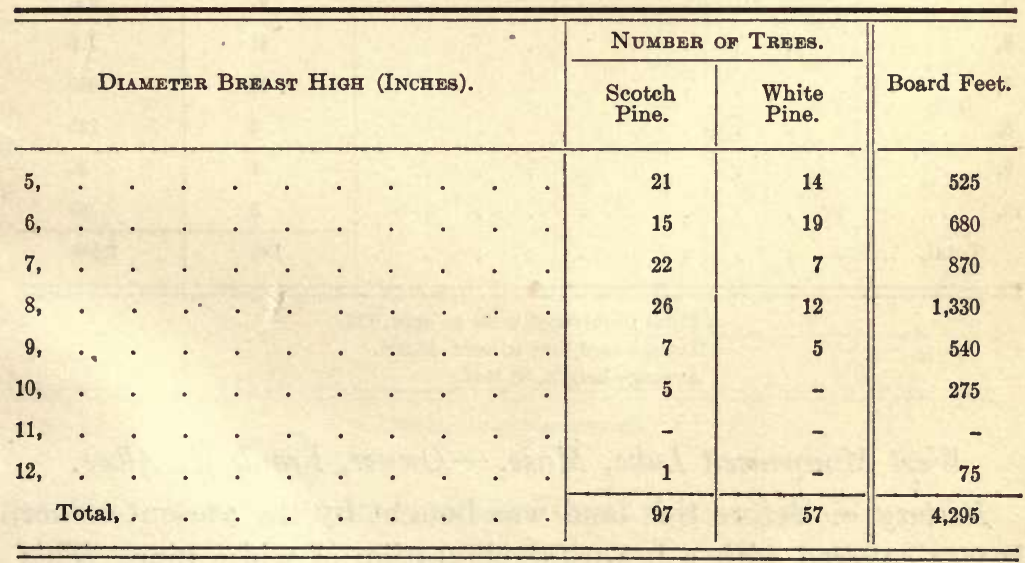

Total number of trees to acre, 616 .

Total board feet to acre, 17,180.

Average height, 40 feet. 
Plot No. 3. Tamarack.

Area, $1 / 4$ acre; age, thirty-eight years.

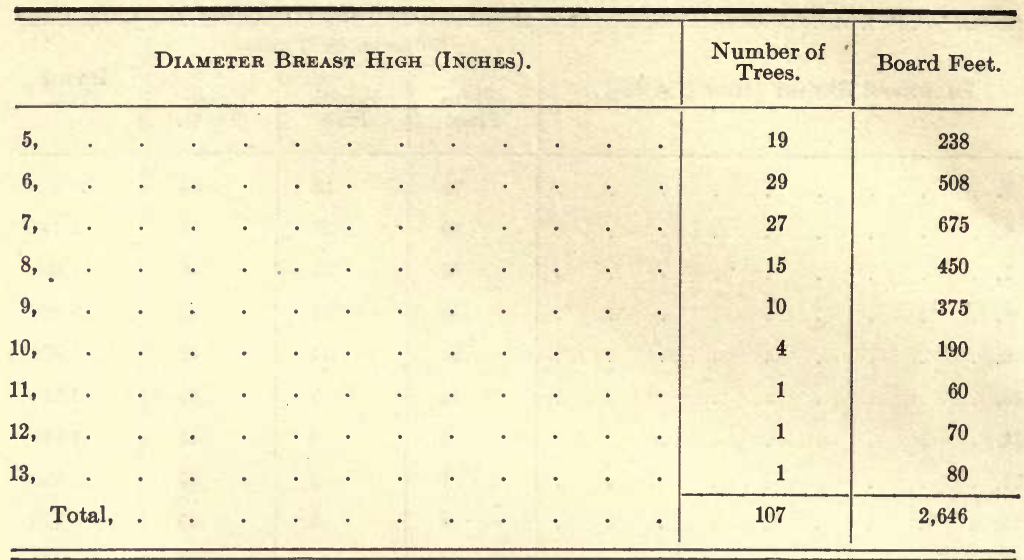

Total number of trees to acre, 428.

Total board feet to acre, 10,584 .

Average height, 35 feet.

Plot No. 4. Tamarack.

Area, $1 / 4$ acre; age; thirty-eight years.

\begin{tabular}{|c|c|c|c|c|c|c|c|c|}
\hline & Diameter & Breas & ST HIGH & (INCHES). & & . & $\begin{array}{l}\text { Number of } \\
\text { Trees. }\end{array}$ & Board Feet. \\
\hline 5. & . & . & . & . & . & . & 92 & 920 \\
\hline 6 , & . & . & . & $\cdot$ & . & . & 51 & 765 \\
\hline 7 , & . & . & . & . & . & . & 32 & 640 \\
\hline 8, & • & . & . & . & . & . & 5 & 125 \\
\hline 9 , & . $\quad$. & . & . & . & . & . & 1 & 30 \\
\hline 10 , & . $\quad$. & . & & . & . & . & 2 & 80 \\
\hline Total, & . . . & . & . & . & . & . & 183 & 2,550 \\
\hline
\end{tabular}

Total number of trees to acre, 732 .

Total board feet to acre, 10,200 .

Average height, 30 feet.

West Monponsett Lake, Mass. - Owner, Frank H. Albee.

History. - Before this land was bought by the present owner it was eovered with a beautiful plantation of white pine. This was for the most part lumbered in 1913 and cut, according to the buyer of the timber, 30,000 board feet to the acre. Measurements have been made of a sample acre of stumps and one inch 
deducted to determine the diameter of the original trees breast high. The plantation was made about forty-six years ago by Samuel Alden, East Bridgewater, Mass., and a Mr. Kingman of Brockton, Mass., and the land selected was on an abandoned farm. Planting was begun in the fall and finished in the spring, and the spacing was done by means of cross-plowed furrows about 10 feet apart.

Objects. - Interest in reforestation, and to utilize waste land.

Treatment. - The removal of dead trees was the only care given this tract, so far as could be determined. The wide spacing prevented excessive overcrowding, but the diameter growth was much better than the height, as estimated from the few trees left standing.

\section{Sample Plot.}

Area, 1 acre; age, forty-six years.

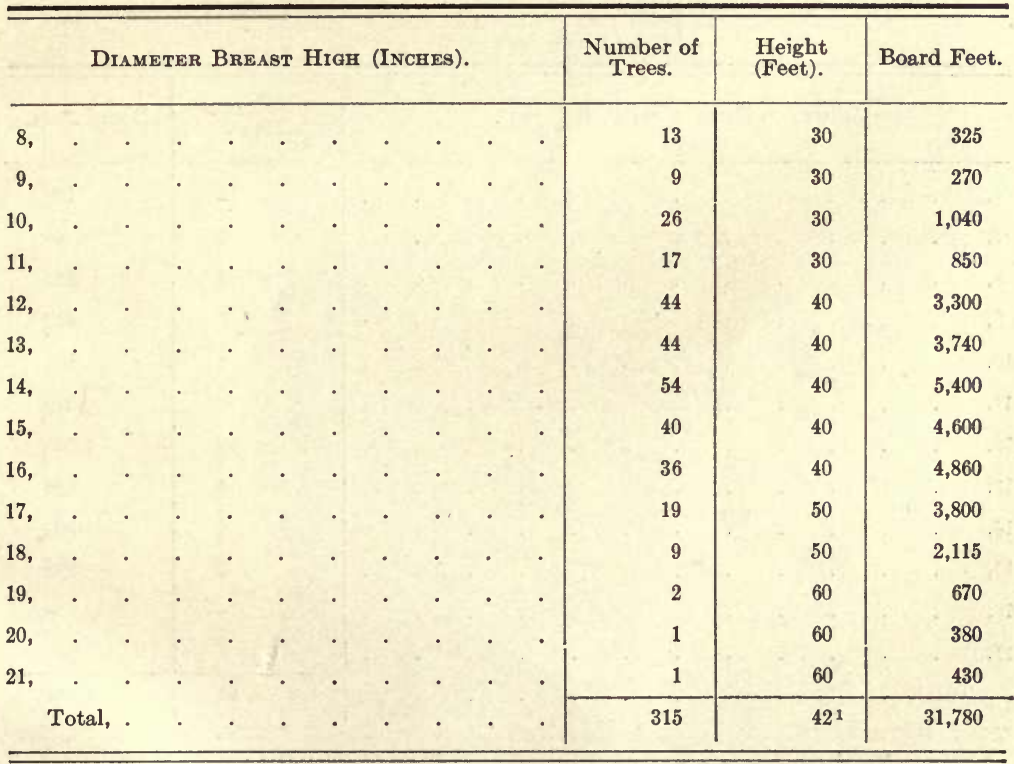

1 Average height estimated.

Rehoboth, Mass. - Owners, Miss Fannie Douse and Mrs. Clara I. Hubbard.

History. - This fifty-five year old plantation was made by Mr. Christopher Carpenter. Pasture trees were used and the spacing was about 8 to 10 feet. At the end of the first ten 
years the trees had reached the height of a man's head. The property is now in the possession of Miss Fannie Douse and Mrs. Clara I. Hubbard.

Treatment. - About four years ago the dead trees were removed and measures taken to protect the plantation from forest fires.

An examination of the tract showed so regular a growth, and so much care in lining up the rows, that a slightly different method was used in measuring it, as follows: -

Two rows of trees through the plantation were measured for heights and diameters, and the contents in each case multiplied by the total number of rows. As a check on this work, a sample quarter acre was measured, with about the same average result.

Row No. 1.

Area, 7 acres; age, fifty-five years.

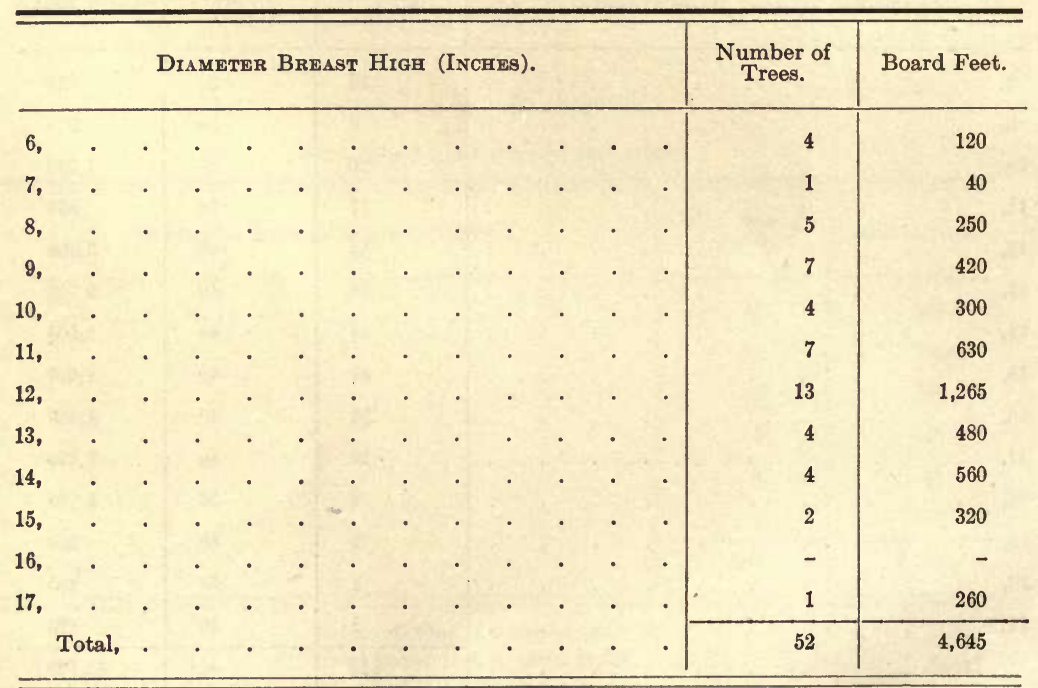

Total number of rows, 66 .

Total board feet, 306,570 .

Total number of acres, 7 .
Total board feet to acre, 43,796 . Average height, 50 feet. 


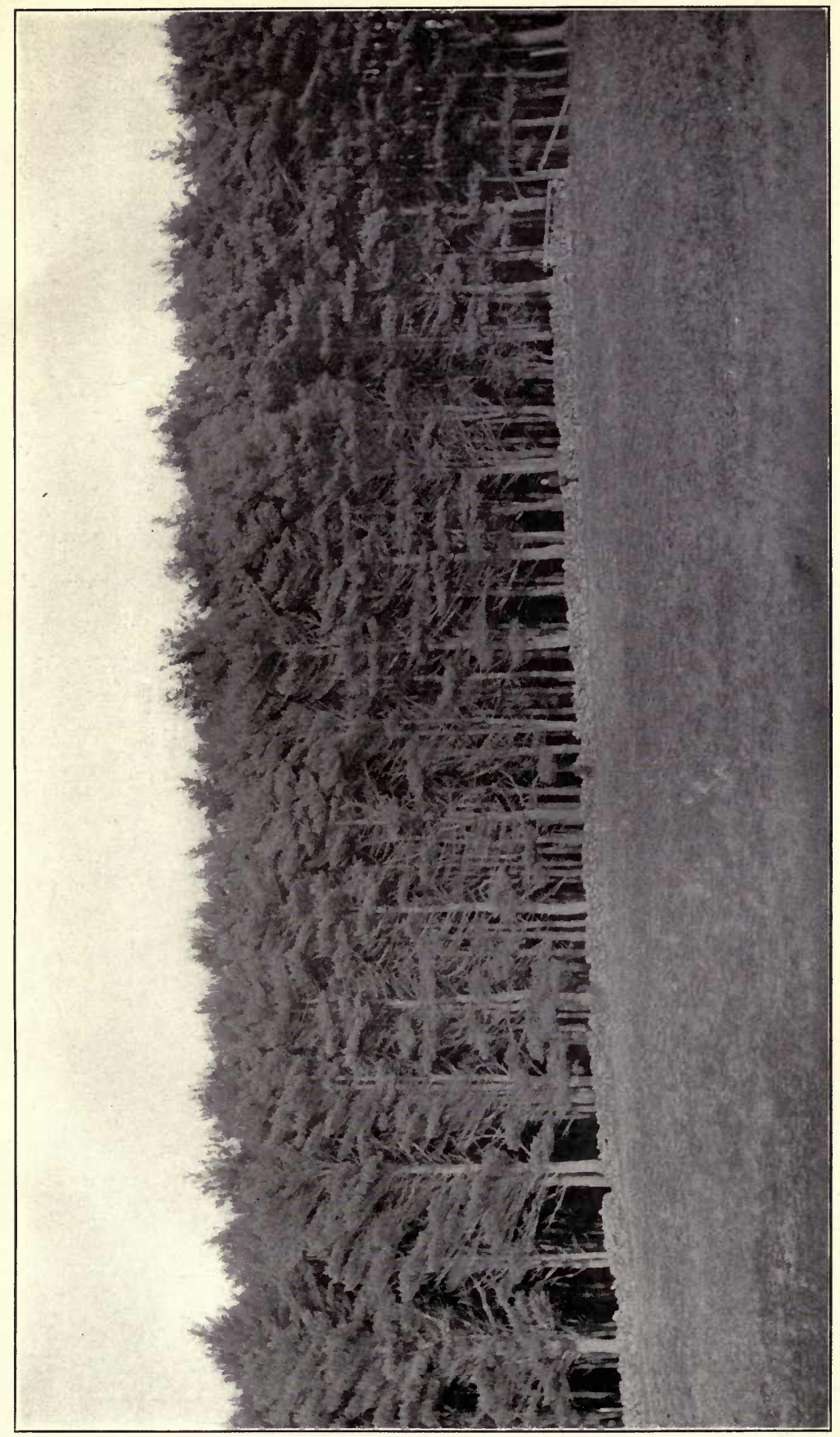

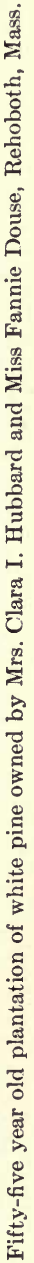






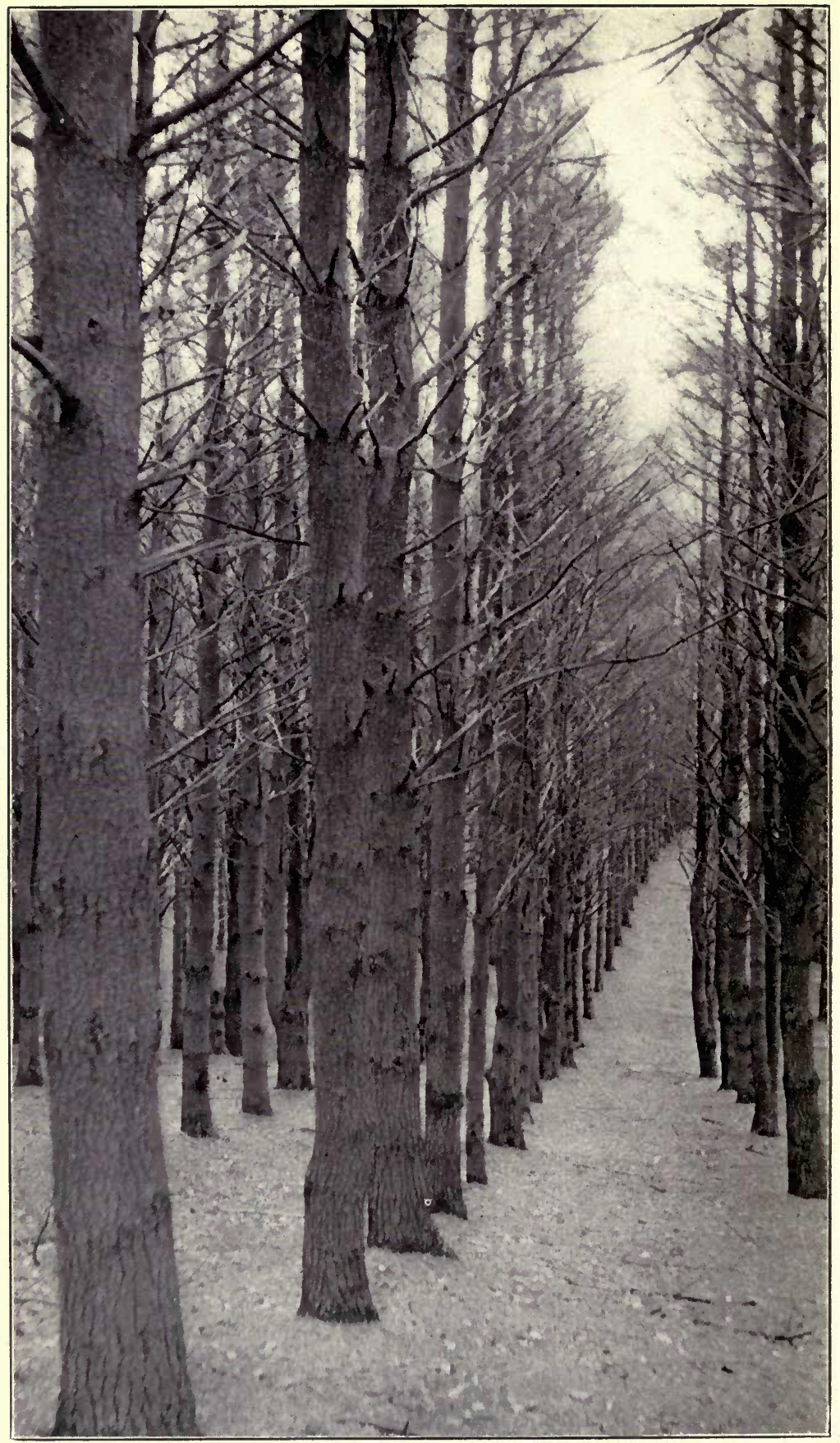

A view of the same tract, taken inside the woods. 
Row No. 2.

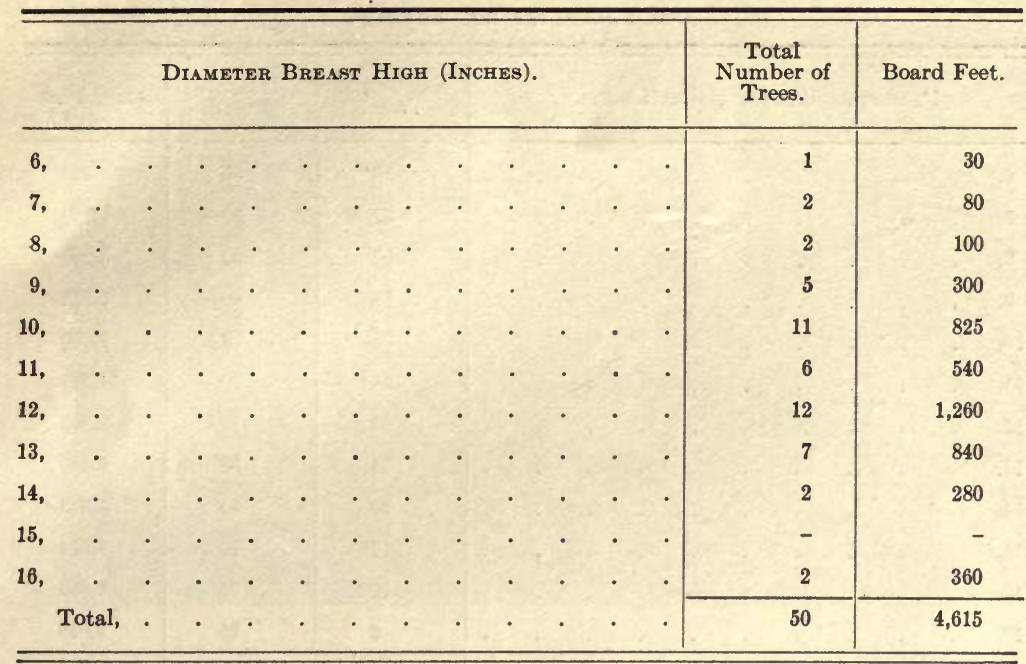

Total number of rows, 66 .

Total board feet, 304,590 .

Total board feet to acre, 43,512.

Total number of acres, 7 .

Average height, 50 feet.

Total Results Averaging Rows Nos. 1 and 2.

Area, 7 acres.

Number of trees to acre, 480.

Board feet to acre, 43,654 .

Sharon, Mass. - Owner, Mr. Edwin Fobes.

History. - The original planter was Mr. Lyman Plimpton, who in 1858 planted white pine on the site of an old peach orchard which had been killed by blight. When first made, the plantation comprised about 6 acres, but a forest fire swept through it a number of years ago, destroying or badly damaging over half of it. A few of the remaining trees show scars upon their trunks, but otherwise appear very thrifty.

Purposes. - To utilize poor land. Interest in forestry, from the standpoint of lumber value and beauty of landscape. 
White Pine.

Area, 2 acres; age, fifty-six years.

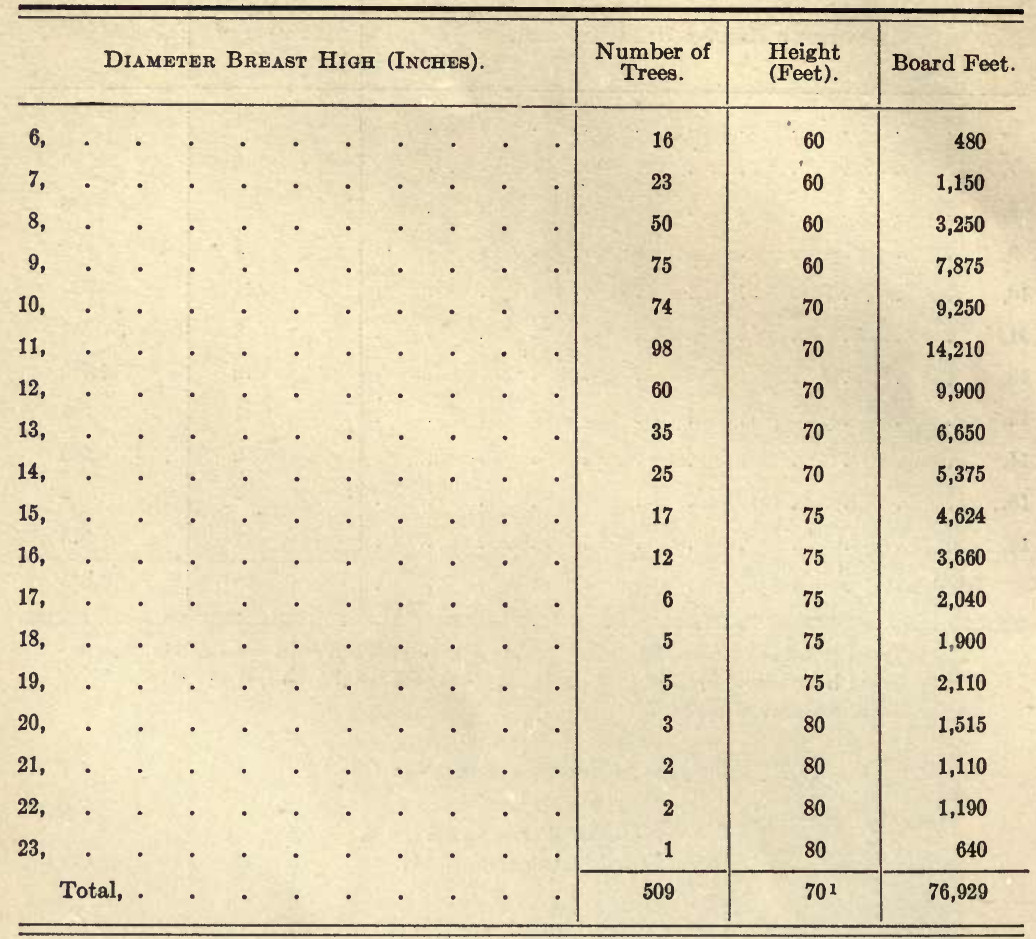

Total number of trees to acre, 254.

Total board feet to acre, 38,464 .

1 Average height.

Along the road which passes this plantation is a row of pine trees which were planted at the same date. Their growth, on account of the added amount of light they have received, has been so much greater than that of average forest trees that it was deemed advisable to measure them separately. While their height is about 10 feet less than that of the adjoining plantation, they show a far greater diameter growth, which will give some idea of what might be expected from white pine if given every possible advantage. 


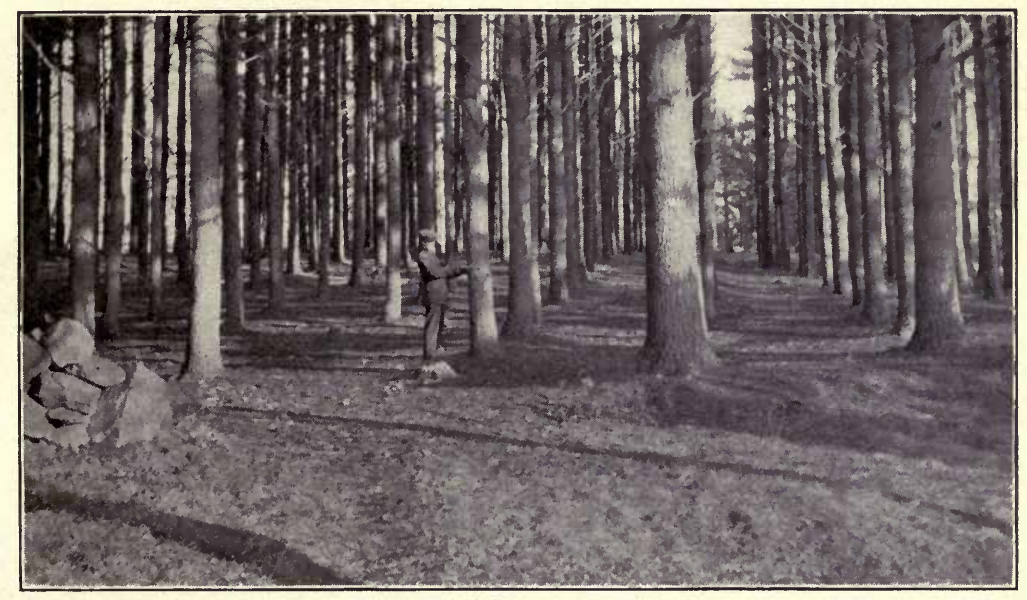

Fifty-six year old plantation of white pine. Edwin Fobes, Sharon, Mass.

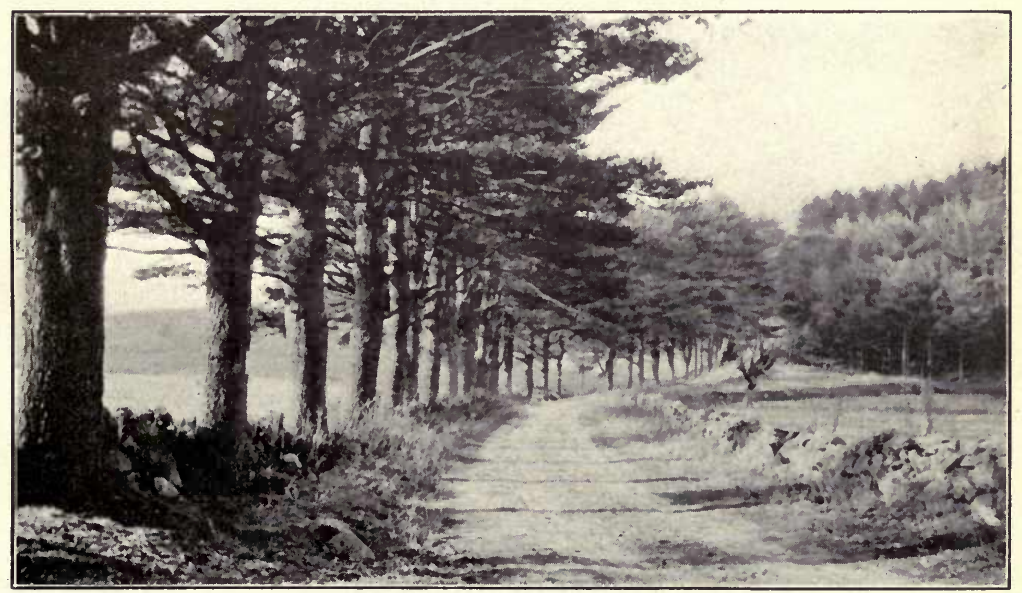

A row of forty-two white pine trees, fifty-six years old. Edwin Fobes plantation, Sharon, Mass. 




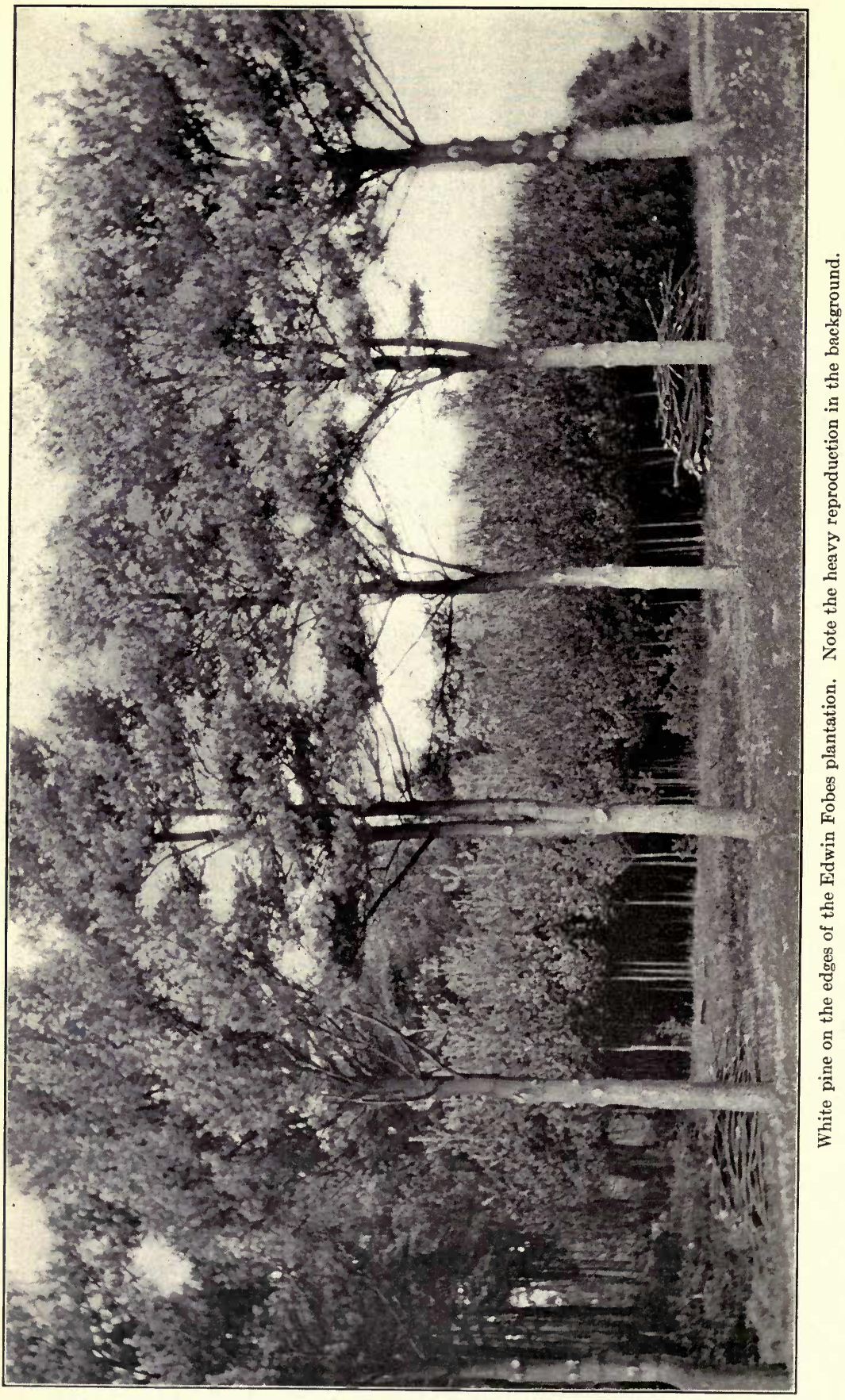


White Pine.

Measurements for a row of trees along road spaced about 10 feet; age, fifty-six years. (See illustration.)

\begin{tabular}{|c|c|c|c|c|c|c|c|c|c|c|}
\hline \multicolumn{9}{|c|}{ Diameter Breast High (Inches). } & \multirow{2}{*}{$\begin{array}{c}\begin{array}{c}\text { Number of } \\
\text { Trees. }\end{array} \\
1\end{array}$} & \multirow{2}{*}{$\frac{\text { Board Feet. }}{200}$} \\
\hline 15 , & & . & . & . & . & . & . & • & & \\
\hline 16, & & - & . & . & . & - & - & - & 2 & $460-$ \\
\hline 17, & & - & . & . & . & . & . & . & - & - \\
\hline 18, & & . & . & . & . & . & . & . & 3 & 885 \\
\hline 19, & & . & . & . & . & . & . & . & 4 & 1,340 \\
\hline 20, & & . & . & . & . & . & . & . & 8 & 3,040 \\
\hline 21, & & . & . & . & . & . & - & . & 6 & 2,880 \\
\hline 22 , & & . & . & . & . & . & . & . & 2 & 1,040 \\
\hline 23, & & . & . & . & . & . & - & - & 5 & 2,825 \\
\hline 24, & & . & . & . & . & . & - & . & 1 & 600 \\
\hline 25, & & . & . & . & . & . & . & - & 4 & 2,580 \\
\hline 26, & & . & . & . & . & . & - & . & 4 & 3,440 \\
\hline 27, & & . & . & . & . & . & . & . & 2 & 1,880 \\
\hline & & . & . & . & . & . & . & . & 42 & 21,170 \\
\hline
\end{tabular}

Average height, 60 feet.

Because these trees are planted in a straight row, 10 feet apart, the spread of the branches, laterally, is nearly 50 feet. It would therefore require only about 80 trees of this size to cover an acre of ground. In terms of the above measurements they would yield a little over 40,000 board feet.

Bridgewater, Mass. - Owner, C. M. Cook.

History. - So far as could be ascertained from residents in the vicinity of this small plantation it was made about fifty years ago, trees dug up from the surrounding pastures being used. Stumps within and on the border of the lot show between 45 and 50 rings. Other information than these observations and the measurements given is lacking. 
White Pine.

Area, 200 by 320 feet (approximately $11 / 3$ acres); age, fifty years.

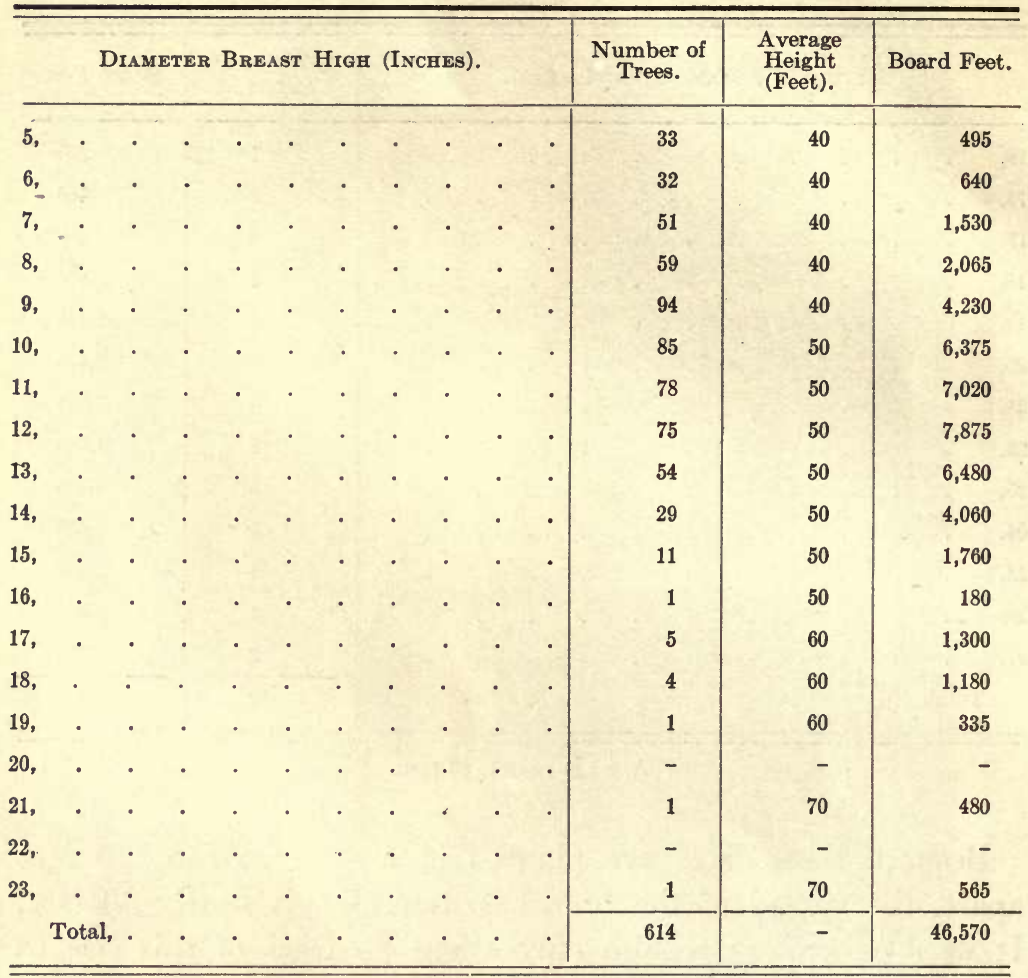

Total number of trees to acre, 460 .

Total board feet to acre, 34,927 .

East Bridgewater, Mass. - Owner, Mr. Harris Latham.

History. - Mr. Latham's plantation is one of the largest investigated, and covers about 12 acres. It can be seen from any one of three roads, and has the appearance of a well-kept park. The original planter was Mr. Galen Latham, father of the present owner, and the trees were set out in 1875, in furrows, plowed 8 to 10 feet apart. Pasture trees 10 to 12 inches in height were used.

Objects. - Interest in forestry. To improve the landscape and to utilize land difficult of successful cultivation. To utilize a large number of seedlings which had sprung up in the pasture. 


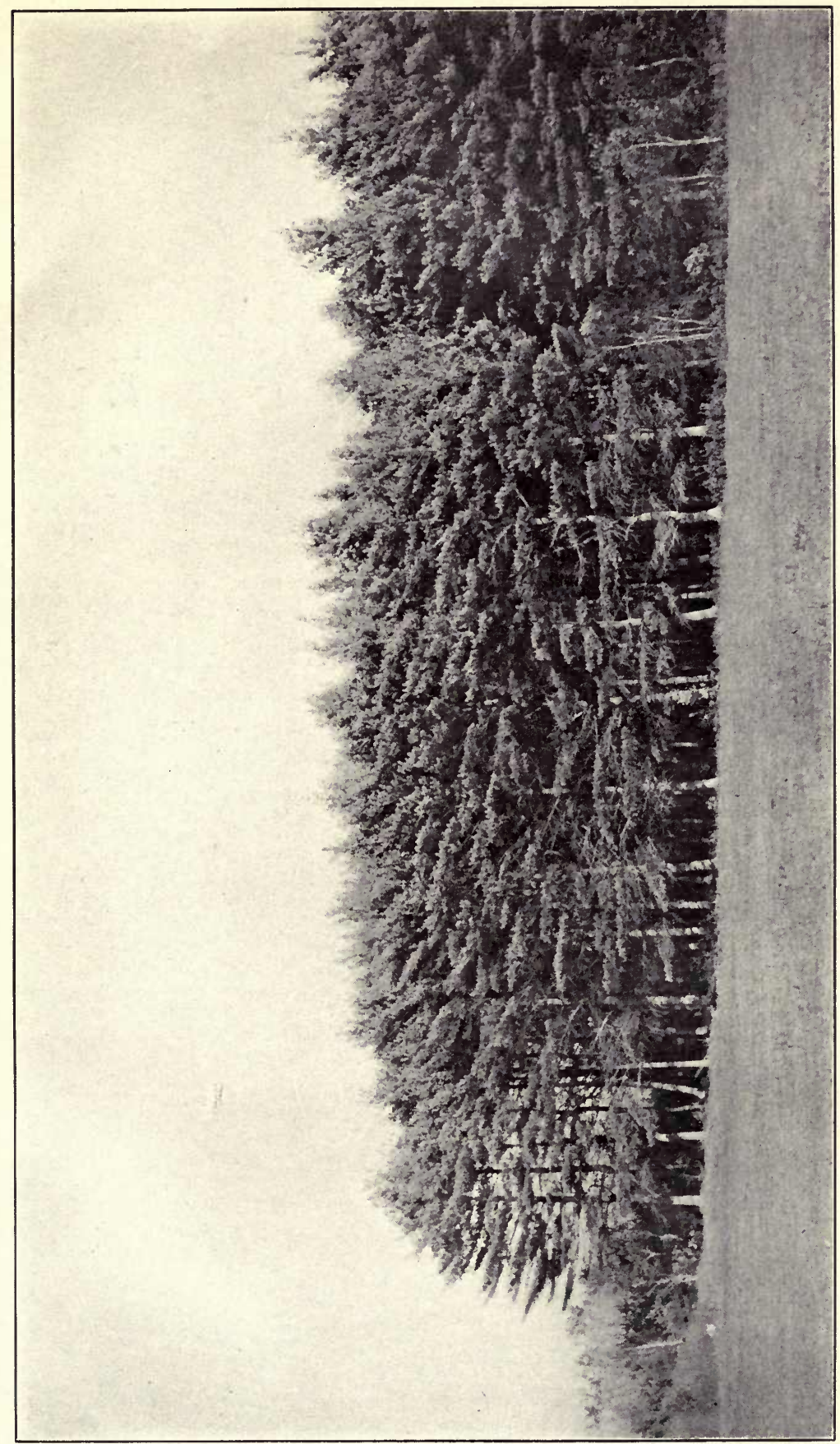

焉 



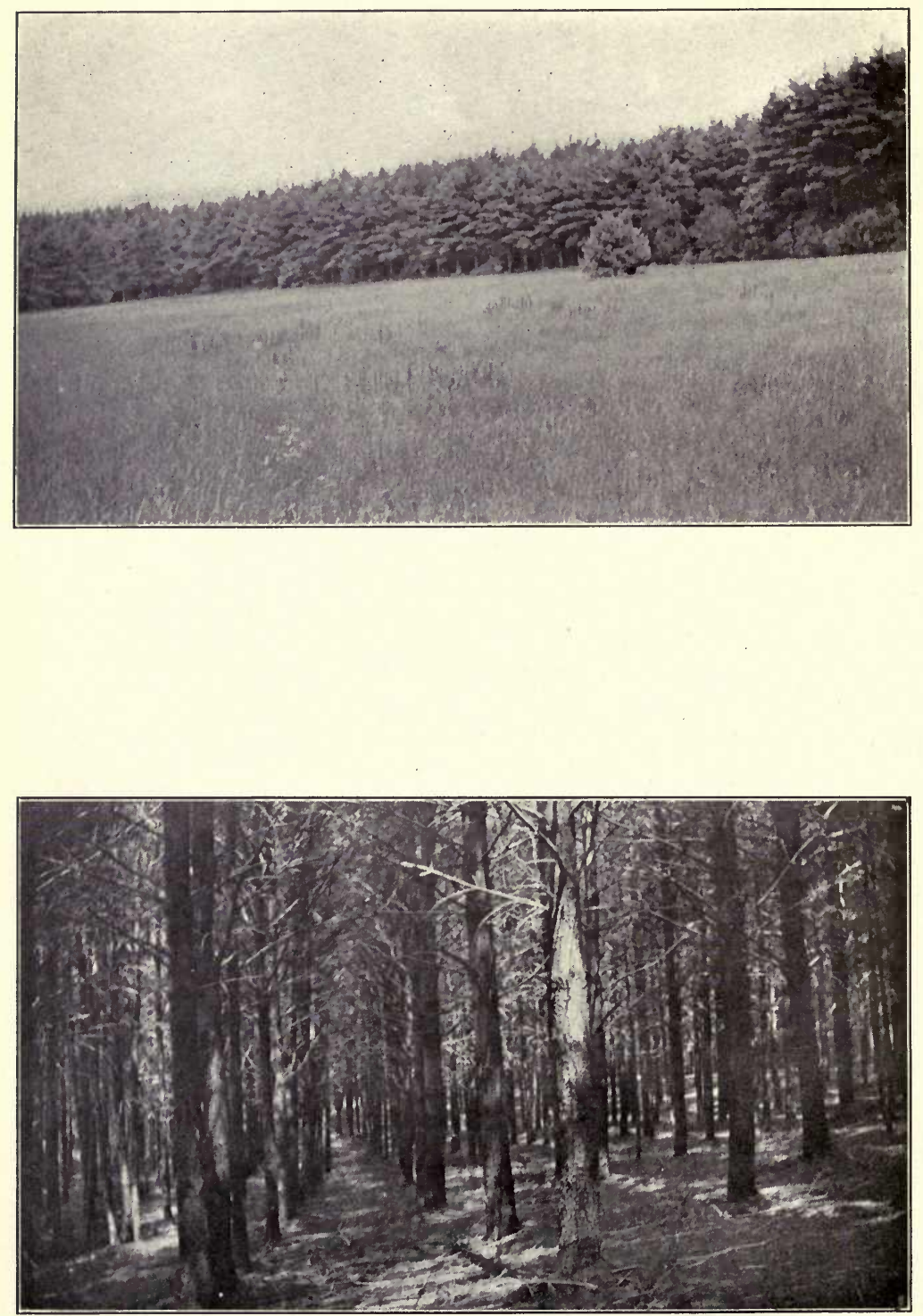

Two views of the Harris Latham plantation, East Bridgewater, Mass.; age, thirty-nine years. 
Treatment. - No thinnings have been made. A very few dead trees have been removed, and no limbs and refuse allowed to remain on the ground.

This experiment is the most extensive recorded in this bulletin from the standpoint of the number of trees measured. The land was resurveyed as a check on the area.

\section{White Pine.}

Area, 12 acres; age, thirty-nine years.

\begin{tabular}{|c|c|c|c|c|c|c|c|c|c|c|}
\hline \multicolumn{9}{|c|}{ Diameter Breast High (Inches). } & \multirow{2}{*}{$\begin{array}{c}\begin{array}{c}\text { Number of } \\
\text { Trees. }\end{array} \\
470\end{array}$} & \multirow{2}{*}{$\begin{array}{r}\text { Board Feet. } \\
4,700\end{array}$} \\
\hline 5, & . & . & - & . & . & . & . $\quad$. & . $\quad$. & & \\
\hline 6 , & . & . & - & . & . & . & . & . & 749 & 22,470 \\
\hline 7, & . & . & - & . & . & . & . & . & 896 & 35,840 \\
\hline 8, & · & . & - & . & . & . & . & . & 981 & 49,050 \\
\hline 9, & . & . & - & . & . & . & . & . & 927 & 55,620 \\
\hline 10, & . & . & . & . & . & . & . & . & 705 & 52,875 \\
\hline 11, & . & . & $\cdot$ & . & . & . & . & . & 371 & 33,390 \\
\hline 12 , & . & . & • & . & . & . & . & . & 170 & 17,850 \\
\hline 13 , & . & . & - & . & . & . & . & . & 85 & 10,200 \\
\hline 14, & . & . & . & . & . & . & . & . & 53 & 7,420 \\
\hline 15, & . & . & - & . & . & . & . & . & 15 & 2,400 \\
\hline 16 , & . & . & . & . & . & . & . & . & 8 & 1,440 \\
\hline \multicolumn{2}{|c|}{ Total, } & . & - & . & . & . & . & . & 5,430 & 293,255 \\
\hline
\end{tabular}

Total number of trees to acre, 452.

Total board feet to acre, 24,428 .

Average height, 50 feet.

South Lancaster, Mass. - Owner, Mr. Harold Parker.

History. - This plantation was made by the father of the present owner forty-three years ago. Natural seedlings were used, taken from the surrounding woods, and spaced about 10 by 10 feet. The whole area reforested covered between 15 and 20 acres, and, besides pine, several types of hardwoods were planted. The area from which measurements were taken, and devoted almost entirely to pine, is about 4 acres.

Objects. - To utilize waste land for commercial advantage, and to determine what might be expected from planted white pine as an investment. To improve the appearance of the land 
from the standpoint of beauty of landscape. Interest in horticulture and the care of woodlands.

Treatment. - In 1905 the government laid out four sample plots, numbered and measured the diameters of all the trees in each plot, and most of the suppressed trees were removed. The total numbers of trees removed (compare following lists of data) were as follows: -

Plot No. 1, Plot No. 2,

Plot No. 3, Plot No. 4,

A record of the data taken in 1905 was loaned by the United States Forest Service in Washington and compared with measurements made in 1914. For convenience in obtaining board measure, trees whose diameters fell over or under the half-inch division of the rule were recorded to the nearest inch. No height measurements were made in 1905 . The heights for that date have therefore been estimated at 5 feet below the measurements for 1914. It is believed that this is a conservative estimate.

Sample Plot No. 1 (Government Plot No. 4).

Area, $1 / 4$ acre; age, forty-three years.

\begin{tabular}{|c|c|c|c|c|c|c|c|c|}
\hline \multirow{2}{*}{ TrEe } & \multirow{2}{*}{ Number. } & \multirow{2}{*}{ Species. } & \multicolumn{2}{|c|}{$\begin{array}{l}\text { DIAMETER } \\
\text { BREAST HIGH } \\
\text { (INCHES). }\end{array}$} & \multirow[t]{2}{*}{ Class. } & \multicolumn{2}{|c|}{ BoARd Feet. } & \multirow{2}{*}{$\begin{array}{c}\text { Gain } \\
\text { in } \\
\text { Board } \\
\text { Feet. }\end{array}$} \\
\hline & & & 1905. & 1914. & & 1905. & 1914. & \\
\hline 1, & . & White pine, & 12 & 12 & 2 & 105 & 120 & 15 \\
\hline 2, . & . & White pine, & 12 & 13 & 1 & 105 & 138 & 33 \\
\hline 3, & . & White pine, & 12 & 12 & 1 & 105 & 120 & 15 \\
\hline 4 , & . & White pine, & 10 & 12 & 1 & 75 & 120 & 45 \\
\hline 5 , & . & White pine, & 10 & 11 & 1 & 75 & $1 \mathbf{Q}^{3}$ & 28 \\
\hline 6 & . & White pine, & 13 & 14 & 1 & 120 & 158 & 38 \\
\hline 7 & . & White pine, - & 10 & 10 & 2 & 75 & 85 & 10 \\
\hline 8, & . & White pine, & 10 & 10 & 2 & 75 & 85 & 10 \\
\hline 9, . & . & White pine, & 13 & 14 & 1 & 120 & 158 & 38 \\
\hline 10 , . & . & White pine, & 13 & 14 & 1 & 120 & 158 & 38 \\
\hline 11, . & . & White pine, & 13 & 15 & 1 & 120 & 180 & 60 \\
\hline 12, & . & White pine, & 15 & 15 & 1 & 160 & 180 & 20 \\
\hline
\end{tabular}




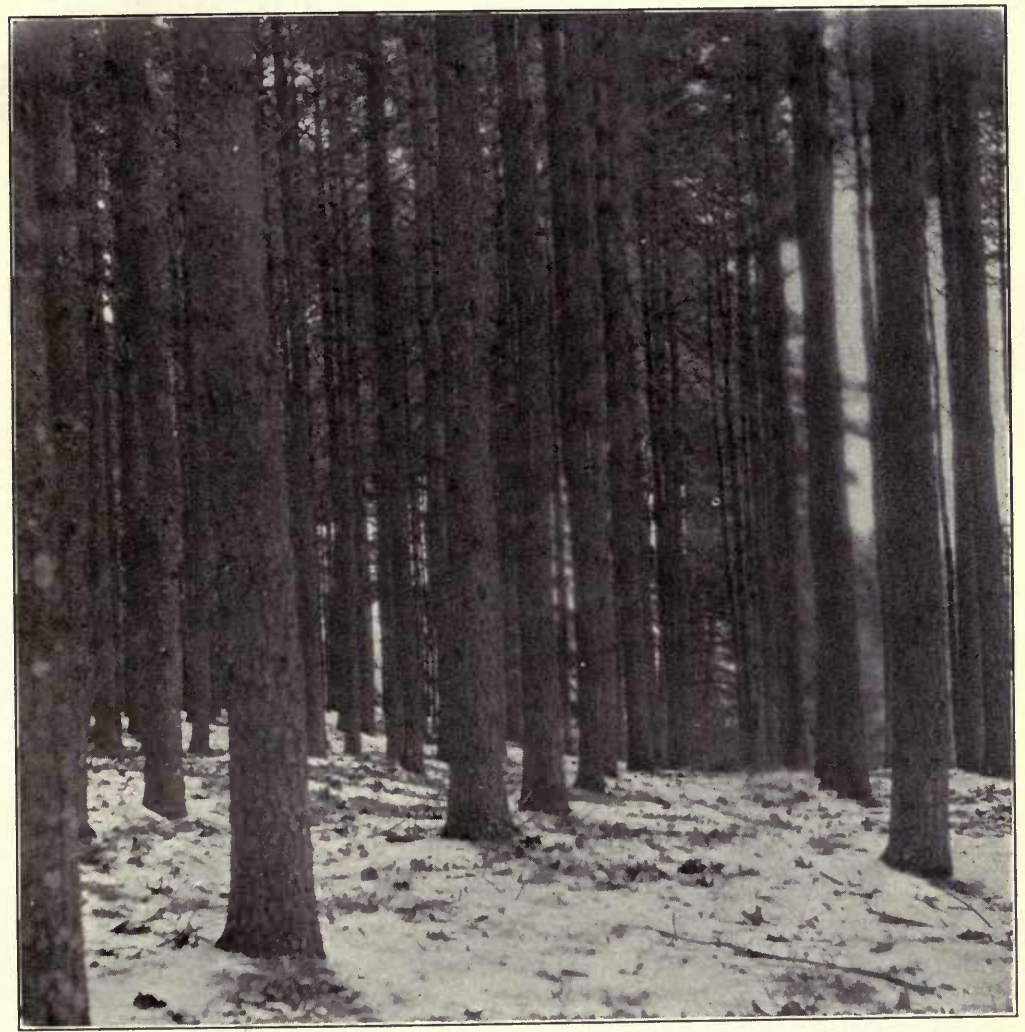

Forty-three year old plantation of white pine which is making a current annual growth of about 1,000 B. F. to the acre. Harold Parker, South Lancaster, Mass. 

Sample Plot No. 1 (Government Plot No. 4) - Continued.

\begin{tabular}{|c|c|c|c|c|c|c|c|c|c|}
\hline \multirow[t]{2}{*}{ Tree } & \multirow[t]{2}{*}{ Number. } & \multirow{2}{*}{\multicolumn{2}{|c|}{ Species. }} & \multicolumn{2}{|c|}{$\begin{array}{c}\text { DIAMETER } \\
\text { BREAST HIGH } \\
\text { (INCHES). } \\
\end{array}$} & \multirow[t]{2}{*}{ Class. } & \multicolumn{2}{|c|}{ Bonrd Feet. } & \multirow{2}{*}{$\begin{array}{c}\text { Gain } \\
\text { in } \\
\text { Board } \\
\text { Feet. }\end{array}$} \\
\hline & & & & 1905. & 1914. & & 1905. & 1914. & \\
\hline 13, & . & White pine, & . & 13 & 14 & 1 & 120 & 158 & 38 \\
\hline 14, & . & White pine, & . & 9 & 9 & 2 & 60 & 70 & 10 \\
\hline 15 , & . & White pine, & . & 12 & 13 & 1 & 105 & 138 & 33 \\
\hline 16, & . & White pine, & . & 10 & 10 & 1 & 75 & 85 & 10 \\
\hline 17, & . & White pine, & . & 12 & 13 & 1 & 105 & 138 & 33 \\
\hline $18,$. & . & White pine, & . & 11 & 12 & 1 & 90 & 120 & 30 \\
\hline 19 , & . & White pine, & . $\quad$. & 10 & 11 & 2 & 75 & 103 & 28 \\
\hline 20, & . & White pine, & . & 10 & 10 & 2 & 75 & 85 & 10 \\
\hline 21, & . & White pine, & . & 10 & 11 & 1 & 75 & 103 & 28 \\
\hline 22, . & . & White pine, & . & 9 & 10 & 2 & 60 & 85 & 25 \\
\hline 23, & . & White pine, & . & 11 & 11 & 1 & 90 & 103 & 13 \\
\hline 24, & . & White pine, & . & 8 & 8 & 2 & 50 & 58 & 8 \\
\hline 25, . & . & White pine, & . & 9 & 10 & 2 & 60 & 85 & 25 \\
\hline 26, & . & White pine, & . & 7 & 7 & 2 & 40 & 45 & 5 \\
\hline 27, & . & White pine, & . & 11 & 12 & 1 & 90 & 120 & 30 \\
\hline 28, & . & White pine, & . & 10 & 11 & 1 & 75 & 103 & 28 \\
\hline 29 , & • & White pine, & . & 12 & 14 & 1 & 105 & 158 & 53 \\
\hline 30 , & . & White pine, & . & 14 & 15 & 1 & 140 & 180 & 40 \\
\hline 31 , & . & White pine, & . & 8 & 8 & 2 & 50 & 58 & 8 \\
\hline 32 , & . & White pine, & . & 11 & 12 & 1 & 90 & 120 & 30 \\
\hline 33, & - & White pine, & . & 11 & 12 & 1 & 90 & 120 & 30 \\
\hline 34 , & . & White pine, & . & 12 & 13 & 1 & 105 & 138 & 33 \\
\hline 35 , & . & White pine, & . & 8 & 8 & 2 & 50 & 58 & 8 \\
\hline 36, & . & White pine, & . & 8 & 8 & 2 & 50 & 58 & 8 \\
\hline 37 , & . & White pine, & . & 12 & 13 & 1 & 105 & 138 & 33 \\
\hline 38, & . & Hemlock, & . & 2 & 3 & 5 & 3 & 3 & - \\
\hline 39, & . & Hemlock, & . & 3 & 4 & 5 & 5 & 5 & - \\
\hline 40, & . & White pine, & . & 13 & 14 & 1 & 120 & 158 & 38 \\
\hline 41, & . & White pine, & . & 8 & 8 & 2 & 50 & 58 & 8 \\
\hline 42, & . & White pine, & . & 10 & 10 & 1 & 75 & 85 & 10 \\
\hline 43, & . & White pine, & . & 8 & 8 & 2 & 50 & 58 & 8 \\
\hline 44, & . & White pine, & . & 14 & 15 & 1 & 140 & 180 & 40 \\
\hline 46 , . & . & White pine, & . & 8 & 8 & 2 & 50 & 58 & 8 \\
\hline 47, . & . & White pine, & . & 11 & 12 & 2 & 90 & 120 & 30 \\
\hline 48, & . & White pine, & . & 12 & 12 & 1 & 105 & 120 & 15 \\
\hline
\end{tabular}


Sample Plot No. 1 (Government Plot No. 4) - Continued.

\begin{tabular}{|c|c|c|c|c|c|c|c|c|c|c|}
\hline \multirow[t]{2}{*}{ Tree } & \multirow{2}{*}{\multicolumn{2}{|c|}{ Number. }} & \multirow{2}{*}{\multicolumn{2}{|c|}{ Species. }} & \multicolumn{2}{|c|}{$\begin{array}{l}\text { DIAMETER } \\
\text { BREAST HIGH } \\
\text { (INCHES). }\end{array}$} & \multirow{2}{*}{ Class. } & \multicolumn{2}{|c|}{ Board Feet. } & \multirow{2}{*}{$\begin{array}{l}\text { Gain } \\
\text { in } \\
\text { Board } \\
\text { Feet. }\end{array}$} \\
\hline & & & & & 1905. & 1914. & & 1905. & 1914. & \\
\hline 49, . & . & . & White pine, & . & 12 & 12 & 2 & 105 & 120 & 15 \\
\hline 50, . & . & . & White pine, & . & 8 & 9 & 1 & 50 & 70 & 20 \\
\hline $51,$. & . & . & White pine, & . & 8 & 8 & 3 & 50 & 58 & 8 \\
\hline 52, . & . & . & White pine, & . & 10 & 11 & 1 & 75 & 103 & 28 \\
\hline 53, . & - & . & White pine, & . & 11 & 12 & 1 & 90 & 120 & 30 \\
\hline $54,$. & . & . & White pine, & . & 12 & 13 & 1 & 105 & 138 & 33 \\
\hline $55,$. & . & . & White pine, & . & 12 & 12 & 1 & 105 & 120 & 15 \\
\hline 56, . & . & . & White pine, & . & 10 & 10 & 1 & 75 & 85 & 10 \\
\hline $57,$. & . & . & White pine, & . & 7 & 7 & 3 & 40 & 45 & 5 \\
\hline 58, . & . & . & White pine, & . & 10 & 11 & 1 & 75 & 103 & 28 \\
\hline 59, . & . & . & White pine, & . & 9 & 10 & 1 & 60 & 85 & 25 \\
\hline 60, . & . & - & White pine, & . & 12 & 13 & 1 & 105 & 138 & 33 \\
\hline 61 , . & . & . & White pine, & . & 10 & 10 & 1 & 75 & 85 & 10 \\
\hline 62, . & . & . & White pine, & . & 12 & 13 & 1 & 105 & 138 & 33 \\
\hline 63, . & . & . & White pine, & . & 10 & 10 & 1 & 75 & 85 & 10 \\
\hline 64, . & . & . & White pine, & . & 14 & 15 & 1 & 140 & 180 & 40 \\
\hline 65, . & . & . & White pine, & . & 11 & 12 & 1 & 90 & 120 & 30 \\
\hline 66, . & . & . & White pine, & . & 12 & 13 & 1 & 105 & 138 & 33 \\
\hline 67, . & . & . & White pine, & . & 12 & 14 & 2 & 105 & 158 & 53 \\
\hline 68, . & . & . & White pine, & . & 13 & 14 & 1 & 120 & 158 & 38 \\
\hline 69, . & . & & White pine, & . & 12 & 13 & 1 & 105 & 138 & 33 \\
\hline 70, . & . & & White pine, & . & 13 & 14 & 1 & 120 & 158 & 38 \\
\hline $71,$. & . & & White pine, & . & 10 & 11 & 1 & 75 & 103 & 28 \\
\hline $72,$. & . & . & White pine, & . & 11 & 11 & 2 & 90 & 103 & 13 \\
\hline $73,$. & . & . & White pine, & . & 9 & 9 & 3 & 60 & 70 & 10 \\
\hline $74,$. & . & . & White pine, & . & 14 & 15 & 1 & 140 & 180 & 40 \\
\hline 75, . & . & . & White pine, & . & 9 & 10 & 2 & 60 & 85 & 25 \\
\hline $76,$. & . & . & White pine, & . & 12 & 13 & 2 & 105 & 138 & 33 \\
\hline $77,$. & . & . & White pine, & . & 13 & 14 & 1 & 120 & 158 & 38 \\
\hline $78,$. & . & . & White pine, & . & 12 & 13 & 1 & 105 & 138 & 33 \\
\hline $79,$. & . & . & White pine, & . & 14 & 16 & 1 & 140 & 205 & 65 \\
\hline 80, . & . & . & White pine, & . & 12 & 14 & 1 & 105 & 158 & 53 \\
\hline 81 , . & . & . & Hemlock, & . & 3 & 3 & 6 & 5 & 5 & - \\
\hline 82, . & . & . & Hemlock, & . & 4 & 4 & 4 & 10 & 10 & - \\
\hline 83, . & . & & White pine, & . & 5 & 5 & 3 & 15 & 25 & 10 \\
\hline
\end{tabular}


Sample Plot No. 1 (Government Plot No. 4) - Concluded.

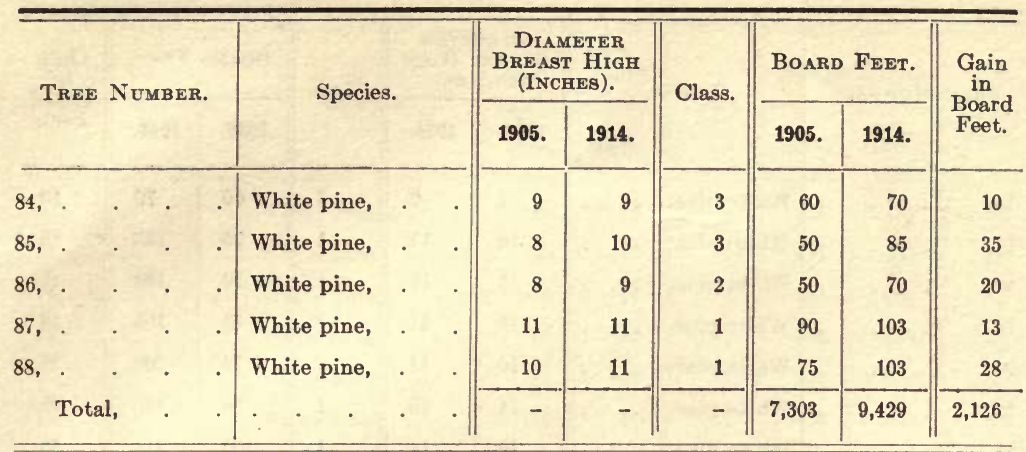

Average height, 1905, 50 feet.

Average height, 1914, 55 feet.

\section{Summary of Data.}

Total board feet to acre, 1905 ,

Total board feet to acre, 1914

Total gain in board feet per acre,

8,504

Current annual gain in board feet per acre,

945

Sample Plot No. 2 (Government Plot No. 5).

Area, $1 / 2$ acre; age, forty-three years.

\begin{tabular}{|c|c|c|c|c|c|c|c|c|c|c|c|c|}
\hline \multirow[t]{2}{*}{ TREE } & \multirow{2}{*}{\multicolumn{3}{|c|}{ NuMBER. }} & \multirow{2}{*}{\multicolumn{2}{|c|}{ Species. }} & & \multicolumn{2}{|c|}{$\begin{array}{l}\text { DIAMETER } \\
\text { BREAST HIGH } \\
\text { (INCHES). }\end{array}$} & \multirow[t]{2}{*}{ Class. } & \multicolumn{2}{|c|}{ Bonrd Feet. } & \multirow{2}{*}{$\begin{array}{l}\text { Gain } \\
\text { in } \\
\text { Board } \\
\text { Feet. }\end{array}$} \\
\hline & & & & & & & 1905. & 1914. & & 1905. & 1914. & \\
\hline 1 , & . & . & - & White pine, & . & - & 13 & 14 & 1 & 120 & 158 & 38 \\
\hline 2 , & - & . & . & White pine, & . & . & 12 & 14 & 1 & 105 & 158 & 53 \\
\hline 3 , & . & - & - & White pine, & . & . & 12 & 14 & 1 & 105 & 158 & 53 \\
\hline 4 , & . & . & . & White pine, & . & . & 11 & 13 & 1 & 90 & 138 & 48 \\
\hline 5 & - & . & . & White pine, & . & . & 8 & 9 & 1 & 50 & 70 & . 20 \\
\hline 6 , & . & . & . & White pine, & . & . & 9 & 11 & 2 & 60 & 103 & 43 \\
\hline 7 & - & - & - & White pine, & - & . & 11 & 13 & 1 & 90 & 138 & 48 \\
\hline 8 & - & . & . & White pine, & . & . & 7 & 9 & 2 & 40 & 70 & 30 \\
\hline 10 & - & . & . & White pine, & . & . & 7 & 9 & 2 & 40 & 70 & 30 \\
\hline 11 , & . & . & - & White pine, & - & . & 6 & 9 & 2 & 30 & 38 & 8 \\
\hline 12 , & - & . & . & White pine, & . & . & 10 & 11 & 1 & 75 & 103 & 28 \\
\hline 13 , & - & - & - & White pine, & - & . & 10 & 11 & 1 & 75 & 103 & 28 \\
\hline 14 & . & - & - & White pine, & - & . & 8 & 9 & 2 & 50 & 70 & 20 \\
\hline 15 & . & . & . & White pine, & . & . & 8 & 8 & 2 & 50 & 58 & 8 \\
\hline
\end{tabular}


Sample Plot No. 2 (Government Plot No. 5) - Continued.

\begin{tabular}{|c|c|c|c|c|c|c|c|c|c|c|c|}
\hline \multirow[t]{2}{*}{ TREE } & \multirow{2}{*}{\multicolumn{3}{|c|}{ NUmber. }} & \multirow{2}{*}{\multicolumn{2}{|c|}{ Species. }} & \multicolumn{2}{|c|}{$\begin{array}{c}\text { DIAMETER } \\
\text { BREAST HIGH } \\
\text { (INCHES). } \\
\end{array}$} & \multirow[t]{2}{*}{ Class. } & \multicolumn{2}{|c|}{ Board Feet. } & \multirow{2}{*}{$\begin{array}{c}\text { Gain } \\
\text { in } \\
\text { Board } \\
\text { Feet. }\end{array}$} \\
\hline & & & & & & 1905. & 1914. & & 1905. & 1914. & \\
\hline 16, & . & . & . & White pine, & . & 9 & 9 & 2 & 60 & 70 & 10 \\
\hline 17, & . & . & . & White pine, & . & 10 & 11 & 1 & 75 & 103 & 28 \\
\hline 18, & - & . & . & White pine, & . & 11 & 11 & 1 & 90 & 103 & 13 \\
\hline 19, & . & . & . & White pine, & . & 10 & 11 & 2 & 75 & 103 & 28 \\
\hline 20 & - & - & . & White pine, & . & 10 & 11 & 2 & 75 & 103 & 28 \\
\hline 22, & - & - & - & White pine, & . & 11 & 12 & 1 & 90 & 120 & 30 \\
\hline 23, & • & . & - & White pine, & . & 12 & 14 & 1 & 10.5 & 158 & 53 \\
\hline 24, & . & . & . & White pine, & : & 9 & 9 & 2 & 60 & 70 & 10 \\
\hline 25, & . & . & . & White pine, & . & 10 & 11 & 1 & 75 & 103 & 28 \\
\hline 27, & • & $\cdot$ & - & White pine, & - & 11 & 13 & 1 & 90 & 138 & 48 \\
\hline 29, & - & . & • & White pine, & - & 8 & 9 & 2 & 50 & 70 & 20 \\
\hline 31, & - & - & - & White pine, & - & 7 & 8 & 2 & 40 & 58 & 18 \\
\hline 33, & . & - & - & White pine, & • & 8 & 8 & 2 & 50 & 58 & 8 \\
\hline 34, & $\cdot$ & - & - & White pine, & - & 6 & 7 & 2 & 30 & 45 & 15 \\
\hline 36, & - & . & - & White pine, & - & 8 & 9 & 2 & 50 & 70 & 20 \\
\hline 43, & $\cdot$ & . & - & White pine, & • & 7 & 7 & 1 & 40 & 45 & 5 \\
\hline 45, & . & . & - & Larch, . & - & 7 & 7 & 1 & 40 & 45 & 5 \\
\hline 46, & . & . & . & White pine, & - & 3 & 3 & 3 & 5 & 8 & 3 \\
\hline 47, & • & . & . & White pine, & · & 4 & 4 & 3 & 10 & 15 & 5 \\
\hline 48, & - & . & - & White pine, & . & 8 & 9 & 2 & 50 & 70 & 20 \\
\hline 49, & . & . & . & White pine, & . & 6 & 6 & 2 & 30 & 38 & 8 \\
\hline 50, & - & · & . & White pine, & - & 6 & 7 & 2 & 30 & 45 & 15 \\
\hline 54, & - & · & . & White pine, & - & 7 & 8 & 2 & 40 & 58 & 18 \\
\hline 55, & - & $\cdot$ & - & White pine, & - & 6 & 7 & 2 & 30 & 45 & 15 \\
\hline 56, & - & - & - & White pine, & - & 6 & 7 & 2 & 30 & 45 & 15 \\
\hline 57, & - & - & - & White pine, & - $\quad \cdot$ & 9 & 10 & 1 & 60 & 85 & 25 \\
\hline 58, & . & $\cdot$ & - & White pine, & - & 8 & 8 & 2 & 50 & 58 & 8 \\
\hline 59, & - & - & . & White pine, & • & 7 & 8 & 2 & 40 & 58 & 18 \\
\hline 60, & - & . & - & White pine, & . & 6 & 7 & 2 & 30 & 45 & 15 \\
\hline 61, & - & . & - & White pine, & . & 9 & 10 & 1 & 60 & 85 & 25 \\
\hline 68, & - & . & • & Larch, & . & 9 & 10 & 1 & 60 & 85 & 25 \\
\hline 69, & . & . & - & Larch, . & . & 5 & 6 & 2 & 15 & 38 & 23 \\
\hline 70, & . & . & • & Larch, . & . & 5 & 5 & 2 & 15 & 25 & 10 \\
\hline 71, & . & . & . & White pine, & . & 12 & 15 & 1 & 120 & 180 & 60 \\
\hline 72, & - & . & • & White pine, & . & 6 & 8 & 3 & 30 & 58 & 28 \\
\hline
\end{tabular}


Sample Plot No. 2 (Government Plot No. 5) - Continued.

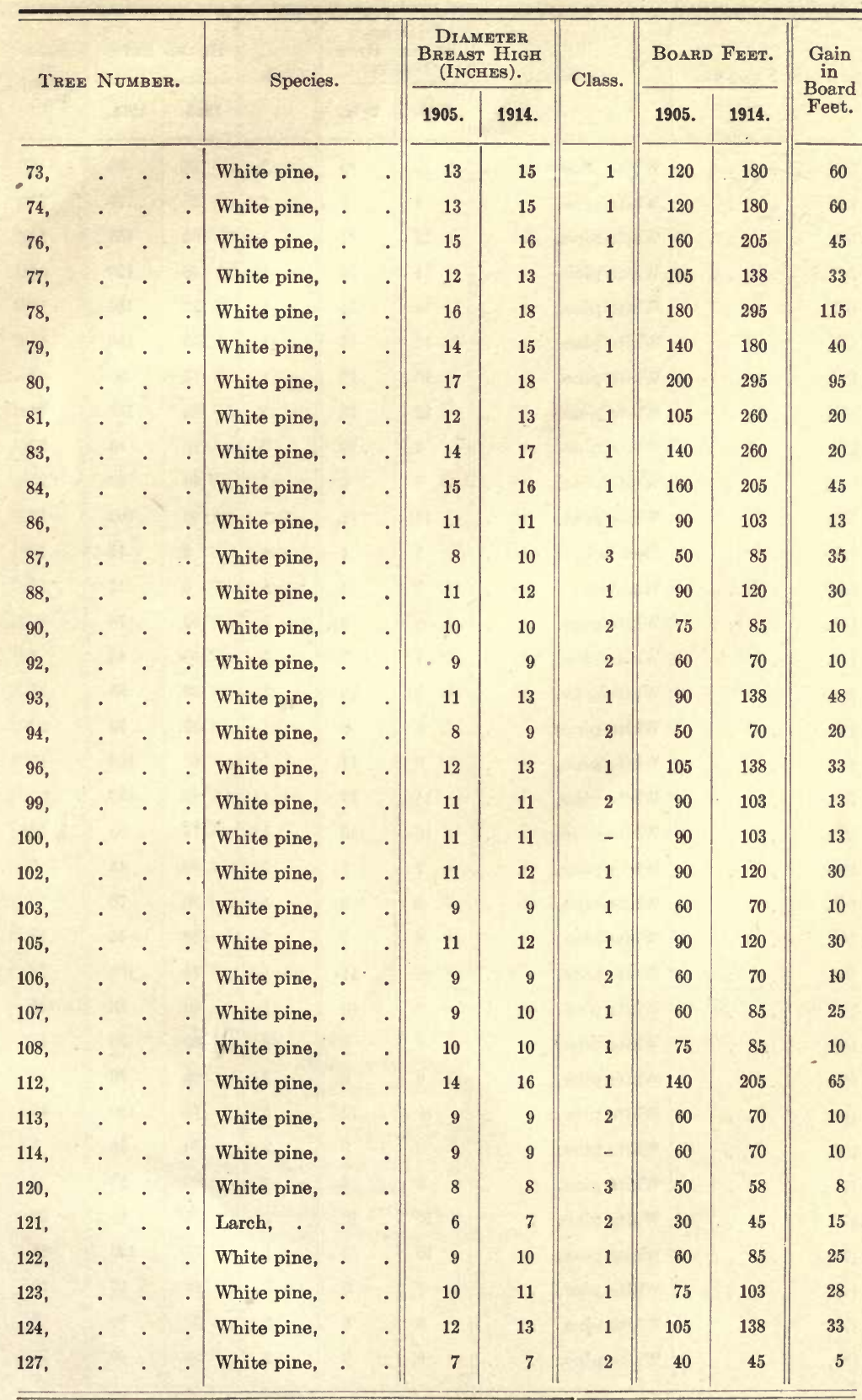


Sample Plot No. 2 (Government Plot No. 5) - Continued.

\begin{tabular}{|c|c|c|c|c|c|c|c|c|c|c|}
\hline \multirow[t]{2}{*}{ TreE } & \multirow{2}{*}{\multicolumn{2}{|c|}{ NUMBER. }} & \multirow{2}{*}{\multicolumn{2}{|c|}{ Species. }} & \multicolumn{2}{|c|}{$\begin{array}{c}\text { Diameter } \\
\text { BREAST HIGH } \\
\text { (INCHES). }\end{array}$} & \multirow[t]{2}{*}{ Class. } & \multicolumn{2}{|c|}{ Bonkd Feet. } & \multirow{2}{*}{$\begin{array}{l}\text { Gain } \\
\text { in } \\
\text { Board } \\
\text { Feet. }\end{array}$} \\
\hline & & & & & 1905. & 1914. & & 1905. & 1914. & \\
\hline 128, & . & - & White pine, & & 10 & 10 & 2 & 75 & 85 & 10 \\
\hline 129, & . & . & White pine, & - & 8 & 9 & 1 & 50 & 70 & 20 \\
\hline 131, & · & - & White pine, & - & 12 & 14 & 1 & 105 & 158 & 53 \\
\hline 132 & · & . & White pine, & . & 11 & 12 & 1 & 90 & 120 & 30 \\
\hline 133, & . & . & White pine, & .. & 13 & 14 & 1 & 120 & 158 & 38 \\
\hline 136, & . & . & White pine, & - & 12 & 14 & 1 & 105 & 158 & 53 \\
\hline 137, & . & . & White pine, & . & 10 & 12 & 1 & 75 & 120 & 45 \\
\hline 138 , & . & . & White pine, & . $\quad$. & 12 & 13 & 1 & 105 & 138 & 33 \\
\hline 144, & . & . & White pine, & . & 9 & 10 & 2 & 60 & 85 & 25 \\
\hline 145, & . & . & White pine, & . & 8 & 9 & 1 & 50 & 70 & 20 \\
\hline 146, & . & . & White pine, & . & 11 & 11 & 1 & 90 & 103 & 13 \\
\hline 147, & . & . & Hemlock, & - & 3 & 4 & 4 & 5 & 15 & 10 \\
\hline 148, & . & . & Hemlock, & - & 3 & 4 & 5 & 5 & 15 & 10 \\
\hline 149 & . & . & White pine, & . & 9 & 9 & 2 & 60 & 70 & 10 \\
\hline 150 , & . & . & White pine, & . & .7 & 7 & 3 & 40 & 45 & 5 \\
\hline 151, & . & . & White pine, & . & 9 & 10 & 1 & 60 & 85 & 25 \\
\hline 152 , & . & . & White pine, & . & 8 & 9 & 1 & 50 & 70 & 20 \\
\hline 154, & . & . & White pine, & - & 9 & 11 & 1 & 60 & 103 & 43 \\
\hline 157, & - & . & White pine, & . & 11 & 12 & 1 & 90 & 120 & 30 \\
\hline 158, & . & . & White pine, & . & 10 & 10 & 1 & 75 & 85 & 10 \\
\hline 159, & . & . & White pine, & . & 7 & 7 & 2 & 40 & 45 & 5 \\
\hline 161 , & . & . & White pine, & . & 8 & 9 & 1 & 50 & 70 & 20 \\
\hline 162 & . & . & White pine, & . & 6 & 7 & 2 & 30 & 45 & 15 \\
\hline 164, & . & . & White pine, & . & 10 & 11 & 1 & 75 & 103 & 28 \\
\hline 166 , & . & . & White pine, & - & 9 & 10 & 1 & 60 & 85 & 25 \\
\hline 167 , & . & . & White pine, & . & 7 & 8 & 2 & 40 & 58 & 18 \\
\hline 168 , & . & . & White pine, & . & 9 & 9 & 2 & 60 & 70 & 10 \\
\hline 169, & . & . & White pine, & - & 10 & 12 & 1 & 75 & 120 & 45 \\
\hline 170, & . & . & White pine, & - & 8 & 8 & 2 & 50 & 58 & 8 \\
\hline 172 . & . & . & White pine, & - & 8 & 8 & 2 & 50 & 58 & 8 \\
\hline 173, & . & - & White pine, & . & 10 & 10 & 2 & 75 & 85 & 10 \\
\hline 174, & - & . & White pine, & - & 10 & 12 & 1 & 75 & 120 & 45 \\
\hline 175, & . & . & White pine, & . & 7 & 8 & 2 & 40 & 50 & 10 \\
\hline 177, & - & . & White pine, & - & 8 & 8 & 2 & 50 & 58 & 8 \\
\hline 178, & . & . & White pine, & - & 8 & 9 & 2 & 50 & 70 & 20 \\
\hline
\end{tabular}


Sample Plot No. 2 (Government Plot No. 5) - Concluded.

\begin{tabular}{|c|c|c|c|c|c|c|c|c|c|c|c|c|}
\hline \multirow[t]{2}{*}{ TREE } & \multirow{2}{*}{\multicolumn{3}{|c|}{ Number. }} & \multirow{2}{*}{\multicolumn{3}{|c|}{ Species. }} & \multicolumn{2}{|c|}{$\begin{array}{c}\text { DIAMETER } \\
\text { BREAST HIGH } \\
\text { (INCHES). } \\
\end{array}$} & \multirow[t]{2}{*}{ Class. } & \multicolumn{2}{|c|}{ Board Feet. } & \multirow{2}{*}{$\begin{array}{c}\text { Gain } \\
\text { in } \\
\text { Board } \\
\text { Feet. }\end{array}$} \\
\hline & & & & & & & 1905. & 1914. & & 1905. & 1914. & \\
\hline 179 , & . & - & - & White pine, & - & . & 11 & 11 & 1 & 90 & 103 & 13 \\
\hline 180 & - & - & - & White pine, & - & . & 8 & 9 & 2 & 50 & 70 & 20 \\
\hline 181 , & . & - & - & White pine, & - & & 9 & 9 & 2 & 60 & 70 & 10 \\
\hline 182, & - & - & - & White pine, & . & . & 9 & 10 & 1 & 60 & 85 & 25 \\
\hline 185 & . & - & - & White pine, & . & . & 6 & 8 & 3 & 30 & 58 & 28 \\
\hline 186 & - & - & . & White pine, & . & - & 11 & 11 & 1 & 90 & 103 & 13 \\
\hline 187, & - & . & . & White pine, & • & . & 8 & 8 & 2 & 50 & 58 & 8 \\
\hline 188 & - & - & - & White pine, & - & - & 10 & 11 & 1 & 75 & 103 & 28 \\
\hline 189 & - & - & . & Larch, & - & - & , & 6 & 2 & 30 & 38 & 8 \\
\hline 191, & - & - & - & White pine, & - & . & 11 & 12 & 1 & 90 & 120 & 30 \\
\hline 193 & - & - & $\bullet$ & White pine, & . & - & 9 & 10 & 1 & 60 & 85 & 25 \\
\hline 195 & - & - & - & White pine, & . & - & 10 & 11 & 1 & 75 & 103 & 28 \\
\hline 196 & - & - & . & White pine, & . & . & 10 & 10 & 1 & 75 & 85 & 10 \\
\hline 197, & - & - & . & White pine, & $\cdot$ & . & 12 & 13 & 1 & 105 & 138 & 33 \\
\hline 199 & $\cdot$ & - & . & White pine, & . & - & 12 & 13 & 1 & 105 & 138 & 33 \\
\hline 200 & - & - & . & White pine, & . & . & 12 & 13 & 1 & 105 & 138 & 33 \\
\hline 201 & - & - & . & White pine, & . & - & 8 & 9 & 1 & 50 & 70 & 20 \\
\hline 202 & - & - & . & White pine, & . & - & 10 & 11 & 1 & 75 & 103 & 28 \\
\hline 203, & . & . & . & White pine, & . & - & 11 & 12 & 1 & 90 & 120 & 30 \\
\hline 204 , & - & . & . & Larch, . & . & . & 9 & 9 & 1 & 60 & 70 & 10 \\
\hline 207 , & - & - & . & Larch, . & - & . & 5 & 6 & 2 & 15 & 38 & 23 \\
\hline 212 , & - & . & . & Larch, . & - & . & 5 & 5 & 2 & 15 & 25 & 10 \\
\hline 213 & . & - & - & Larch, . & - & • & 6 & 6 & 2 & 30 & 38 & 8 \\
\hline 215 & . & - & . & White pine, & - & - & 11 & 13 & 1 & 90 & 138 & 48 \\
\hline Tota & & - & . & $\cdot \quad \cdot$ & - & $\cdot$ & - & - & - & 9,820 & 13,371 & 3,551 \\
\hline
\end{tabular}

Average height, 1905, 50 feet.

Average height, 1914, 55 feet.

Summary of Data.

Total board feet to acre, 1905, . . . . . . 19,640

Total board feet to acre, 1914, . . . . . . 26,742

Total gain in board feet per acre, . . . . . 7,102

Current annual gain in board feet per acre, . $\quad$. $\quad$. 789 
Sample Plot No. 3 (Government Plot No. 6).

Area, $1 / 8$ acre; age, forty-three years.

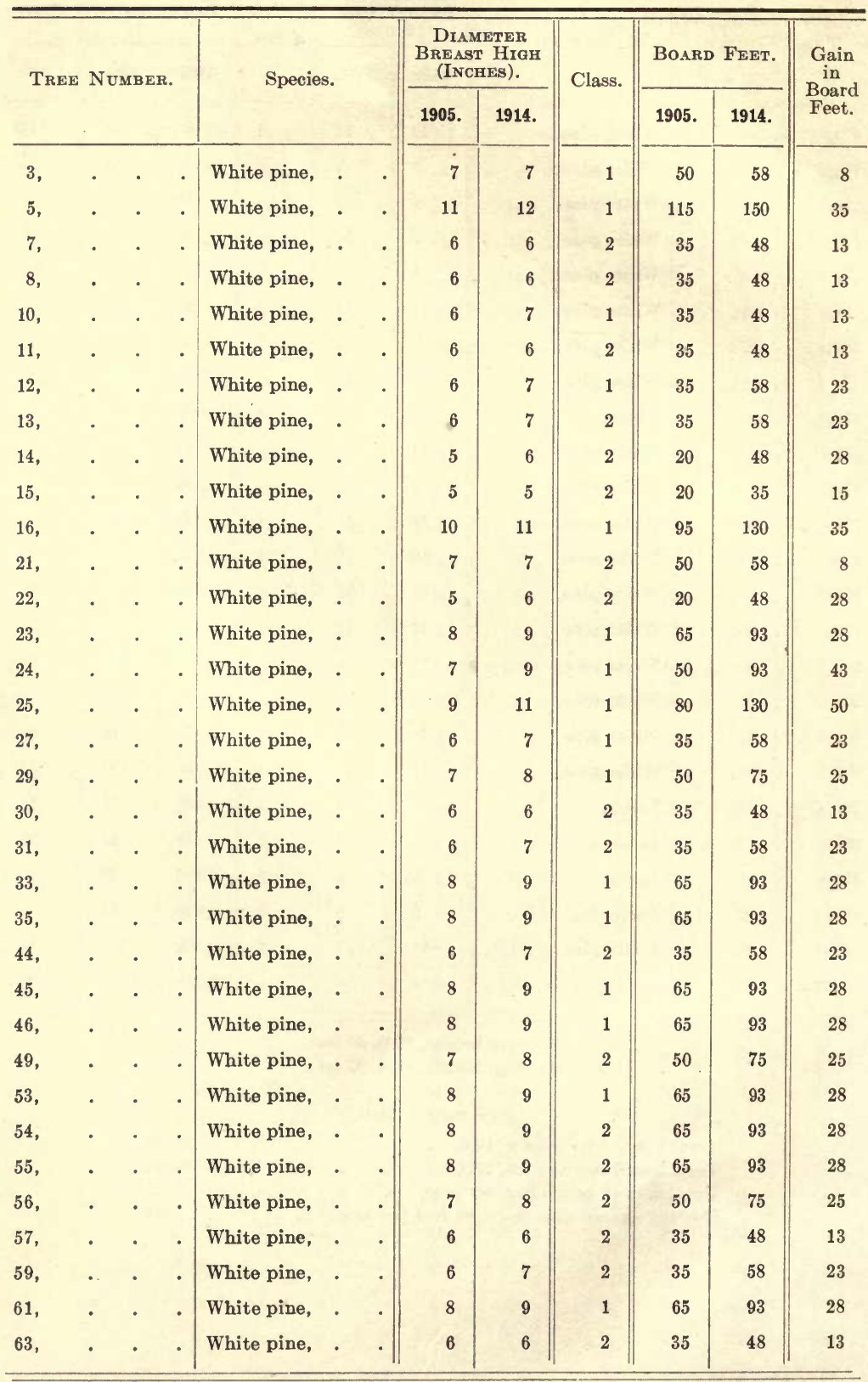


Sample Plot No. 3 (Government Plot No.-6) - Concluded.

\begin{tabular}{|c|c|c|c|c|c|c|c|c|c|c|c|}
\hline \multirow{2}{*}{ TREE } & \multirow{2}{*}{\multicolumn{3}{|c|}{ NUMBer. }} & \multirow{2}{*}{\multicolumn{2}{|c|}{ Species. }} & \multicolumn{2}{|c|}{$\begin{array}{l}\text { DIAMETER } \\
\text { BREAST HIGH } \\
\text { (INCHES). }\end{array}$} & \multirow{2}{*}{ Class. } & \multicolumn{2}{|c|}{ Bonrd Feet. } & \multirow{2}{*}{$\begin{array}{c}\text { Gain } \\
\text { in } \\
\text { Board } \\
\text { Feet. }\end{array}$} \\
\hline & & & & & & 1905. & 1914. & & 1905. & 1914. & \\
\hline 64 , & . & . & . & White pine, & . $\quad$. & 5 & 7 & 2 & 20 & 58 & 38 \\
\hline 66 , & . & . & . & White pine, . & . $\quad$. & 7 & 7 & 2 & 50 & 58 & 8 \\
\hline 67, & . & . & . & White pine, & .. & 8 & 9 & 3 & 65 & 93 & 28 \\
\hline 69 , & . & . & . & White pine, & - . & 7 & 8 & 1 & 50 & 75 & 25 \\
\hline 71, & . & . & . & White pine, & .. & 9 & 10 & 1 & 80 & 110 & 30 \\
\hline 73 & . & . & . & White pine, & · & 9 & 10 & 1 & 80 & 110 & 30 \\
\hline 74, & . & . & . & White pine, & · & 8 & 9 & 2 & 65 & 93 & 28 \\
\hline 75 , & . & . & - & White pine, & .. & 7 & 8 & 2 & 50 & 75 & 25 \\
\hline 76, & . & . & . & White pine, & . $\quad$. & 6 & 6 & 3 & 35 & 48 & 13 \\
\hline 77, & . & . & . & White pine, & . & 9 & 10 & 1 & 80 & 110 & 30 \\
\hline 78, & . & . & • & White pine, & · & 7 & 7 & 2 & 50 & 58 & 8 \\
\hline 81, & . & . & . & White pine, & $\cdot \cdot$ & 9 & 9 & 1 & 80 & 83 & 13 \\
\hline 83, & . & . & . & White pine, & . & 7 & 7 & 2 & 50 & 58 & 8 \\
\hline 85 , & . & . & . & White pine, & • & 6 & 7 & 2 & 35 & 58 & 23 \\
\hline 86 , & . & . & • & White pine, & • & 8 & 10 & 1 & 65 & 110 & 45 \\
\hline 87, & · & . & . & White pine, & • & 5 & 5 & 2 & 20 & 35 & 15 \\
\hline 89 , & . & . & • & Wnite pine, & • & 7 & 7 & 2 & 50 & 58 & 8 \\
\hline 91, & . & . & . & White pine, & • & 8 & 9 & 1 & 65 & 93 & 28 \\
\hline 92, & . & . & - & White pine, & • & 7 & 7 & 2 & 50 & 58 & 8 \\
\hline 94, & . & . & . & White pine, & • & 7 & 7 & 2 & 50 & 58 & 8 \\
\hline 101, & . & . & . & White pine, & . & 9 & 11 & 1 & 80 & 130 & 50 \\
\hline Tota & & . & . &.$\quad \cdot \quad \cdot$ & · & - & - & - & 2,860 & 4,135 & 1,275 \\
\hline
\end{tabular}

Average height, 1905, 60 feet.

Average height, 1914, 65 feet.

Summary of Data.

Total board feet per acre, 1905, . . . . . . . 22,880

Total board feet per acre, 1914 , . . . . . . 33,080

Total gain in board feet per acre, . . . . . 10,200

Current annual gain in board feet per acre, . . . 1,133 
Sample Plot No. 4 (Government Plot No. 7).

Area, $1 / 8$ acre; age, forty-three years.

\begin{tabular}{|c|c|c|c|c|c|c|c|c|c|c|c|c|}
\hline \multirow[t]{2}{*}{ TREE } & \multirow{2}{*}{\multicolumn{3}{|c|}{ NuMBER. }} & \multirow{2}{*}{\multicolumn{3}{|c|}{ Species. }} & \multicolumn{2}{|c|}{$\begin{array}{l}\text { DIAMETER } \\
\text { BREAST HIGH } \\
\text { (INCHES). }\end{array}$} & \multirow{2}{*}{ Class. } & \multicolumn{2}{|c|}{ BonRd FeEt. } & \multirow{2}{*}{$\begin{array}{l}\text { Gain } \\
\text { in } \\
\text { Board } \\
\text { Feet. }\end{array}$} \\
\hline & & & & & & & 1905. & 1914. & & 1905. & 1914. & \\
\hline 1, & . & - & - & White pine, & - & . & 6 & 7 & 2 & 35 & 58 & 23 \\
\hline 2 , & . & . & . & White pine, & - & . & 6 & 6 & 2 & 35 & 48 & 13 \\
\hline 3 & . & - & . & White pine, & . & & 6 & 6 & 2 & 35 & 48 & 13 \\
\hline 5 , & - & . & . & White pine, & - & . & 6 & 6 & 2 & 35 & 48 & 13 \\
\hline 7 & . & . & . & White pine, & . & . & 7 & 8 & 1 & 50 & 75 & 25 \\
\hline 8, & . & . & . & White pine, & - & . & 5 & 5 & 2 & 20 & 35 & 15 \\
\hline 9, & . & . & . & White pine, & - & - & 7 & 8 & 1 & 50 & 75 & 25 \\
\hline 10 & - & . & . & White pine, & - & . & 5 & 5 & 2 & 20 & 35 & 15 \\
\hline 12 , & - & · & - & White pine, & - & - & 7 & 7 & 1 & 50 & 58 & 8 \\
\hline 13 & . & . & . & White pine, & - & - & 6 & 6 & 1 & 35 & 58 & 23 \\
\hline 16, & . & - & . & White pine, & - & . & 6 & 6 & 1 & 35 & 48 & 13 \\
\hline 17 , & . & - & . & White pine, & - & . & 6 & 6 & 2 & 35 & 48 & 13 \\
\hline 18, & . & . & . & White pine, & - & . & 7 & 7 & 1 & 50 & 58 & 8 \\
\hline 19 , & . & . & . & White pine, & . & . & 5 & 5 & 2 & 20 & 35 & 15 \\
\hline 20 & - & . & . & White pine, & - & . & 6 & 6 & 2 & 35 & 48 & 13 \\
\hline 21, & - & : & - & White pine, & - & - & 4 & 5 & 3 & 10 & 35 & 25 \\
\hline 22 , & - & . & . & White pine, & - & . & 7 & 7 & 1 & 50 & 58 & 8 \\
\hline 23 & . & . & . & White pine, & - & . & 8 & 10 & 1 & 65 & 110 & 45 \\
\hline 24, & - & . & . & White pine, & - & - & 5 & 6 & 2 & 20 & 48 & 28 \\
\hline 25 , & . & . & - & White pine, & - & - & 7 & 7 & 2 & 50 & 58 & 8 \\
\hline 26 , & . & . & - & White pine, & - & - & 7 & 8 & 1 & 50 & 75 & 25 \\
\hline 27 , & . & . & - & White pine, & - & - & 6 & 6 & 2 & 35 & 48 & 13 \\
\hline 29 , & . & - & . & White pine, & - & - & 7 & 7 & 2 & 50 & 58 & 8 \\
\hline 30 & . & - & - & White pine, & - & . & 8 & 9 & 1 & 65 & 93 & 28 \\
\hline 35 , & . & $\cdot$ & . & White pine, & - & . & 5 & 5 & 2 & 20 & 35 & 15 \\
\hline 36, & . & . & - & White pine, & - & $\cdot$ & 4 & 4 & 3 & 10 & 15 & 5 \\
\hline 37, & . & . & . & White pine, & - & - & 7 & 8 & 1 & 50 & 75 & 25 \\
\hline 38, & . & . & . & White pine, & • & · & 6 & 7 & 1 & 35 & 58 & 23 \\
\hline 39 , & . & . & - & White pine, & . & $\cdot$ & 4 & 5 & 2 & 10 & 35 & 25 \\
\hline 41, & - & . & - & White pine, & - & - & 7 & 7 & 1 & 50 & 58 & 8 \\
\hline 42 & - & - & - & White pine, & . & - & 5 & 5 & 2 & 20 & 35 & 15 \\
\hline 43 & - & - & . & White pine, & : & - & 6 & 6 & 2 & 35 & 48 & 13 \\
\hline 46 & . & . & - & White pine, & - & - & 7 & 8 & 1 & 35 & 75 & 40 \\
\hline 48, & . & - & . & White pine, & . & - & 5 & 5 & 2 & 20 & 35 & 15 \\
\hline
\end{tabular}


Sample Plot No. 4 (Government Plot No. 7) - Continued.

\begin{tabular}{|c|c|c|c|c|c|c|c|c|c|c|c|}
\hline \multirow[t]{2}{*}{ TrEe } & \multirow{2}{*}{\multicolumn{3}{|c|}{ NUMber. }} & \multirow{2}{*}{\multicolumn{2}{|c|}{ Species. }} & \multicolumn{2}{|c|}{$\begin{array}{c}\text { DIAMETER. } \\
\text { BREAST HIGH } \\
\text { (INCHES). } \\
\end{array}$} & \multirow[t]{2}{*}{ Class. } & \multicolumn{2}{|c|}{ Bontd Feet. } & \multirow{2}{*}{$\begin{array}{c}\text { Gain } \\
\text { in } \\
\text { Board } \\
\text { Feet. }\end{array}$} \\
\hline & & & & & & 1905. & 1914. & & 1905. & 1914. & \\
\hline 49, & . & . & . & White pine, & . & 8 & 9 & 1 & 65 & 93 & 28 \\
\hline 51, & - & . & & White pine, & . & 8 & 9 & 1 & 65 & 93 & 28 \\
\hline 52, & . & . & & White pine, & . & 7 & 7 & 2 & 50 & 58 & 8 \\
\hline 53, & . & . & • & White pine, & . & 7 & 8 & 1 & 50 & 75 & 25 \\
\hline 55 & . & . & • & White pine, & . & 7 & 7 & 2 & 50 & 58 & 8 \\
\hline 56, & . & . & • & White pine, & . & 8 & 9 & 1 & 65 & 93 & 28 \\
\hline 57, & . & . & & White pine, & . & 4 & 4 & 3 & 10 & 15 & 5 \\
\hline 58, & . & . & . & White pine, & . & 4 & 4 & 3 & 10 & 15 & 5 \\
\hline 59 , & . & . & . & White pine, & . & 9 & 9 & 1 & 80 & 93 & 13 \\
\hline 60, & . & . & . & White pine, & . & 6 & 6 & 2 & 35 & 48 & 13 \\
\hline 61 , & . & . & . & White pine, & . & 7 & 7 & 2 & 50 & 58 & 8 \\
\hline 62 , & . & . & . & White pine, & . & 5 & 6 & 3 & 20 & 48 & 28 \\
\hline 63 , & . & . & . & White pine, & . & 4 & 5 & 2 & 10 & 35 & 25 \\
\hline 64, & . & . & . & White pine, & . & 5 & 6 & 2 & 20 & 48 & 28 \\
\hline 65 , & . & . & . & White pine, & . & 7 & 9 & 1 & 50 & 93 & 43 \\
\hline 66, & . & . & . & White pine, & . & 6 & 6 & 2 & 35 & 48 & 13 \\
\hline 68 , & . & . & . & White pine, & . & 6 & $7^{\circ}$ & 2 & 35 & 58 & 23 \\
\hline 70 , & . & . & . & White pine, & - & 7 & 8 & 2 & 50 & 75 & 25 \\
\hline 71 , & . & . & . & White pine, & . & 7 & 7 & 1 & 50 & 58 & 58 \\
\hline 72 , & . & . & . & White pine, & & 10 & 11 & 1 & 95 & 130 & 3 \\
\hline 73, & . & . & . & White pine, & . & 5 & 5 & 2 & 20 & 35 & 15 \\
\hline 74 , & . & . & . & White pine, & . & 5 & 5 & 2 & 20 & 35 & 15 \\
\hline 75, & . & . & • & White pine, & . & 6 & 6 & 2 & 35 & 48 & 13 \\
\hline 76 , & . & . & . & White pine, & . & 6 & 6 & 3 & 35 & 38 & 13 \\
\hline 77, & . & . & . & White pine, & . & 9 & 11 & 1 & 80 & 130 & 50 \\
\hline 79 , & . & . & . & White pine, & · & 8 & 9 & 1 & 65 & 93 & 28 \\
\hline 81 , & . & . & • & White_pine, & . & 5 & 5 & 2 & 20 & 35 & 15 \\
\hline 82 , & . & . & • & White pine, & • & 8 & 8 & 2 & 65 & 75 & 10 \\
\hline 83, & . & . & $\cdot$ & White pine, & & 5 & 6 & 2 & 20 & 48 & 28 \\
\hline 85, & . & . & - & White pine, & . & 7 & 8 & 2 & 50 & 75 & 25 \\
\hline 86, & . & . & • & White pine, & . & 8 & 8 & 1 & 65 & 75 & 10 \\
\hline 87, & . & . & $\cdot$ & White pine, & . & 7 & 7 & 2 & 50 & 58 & 8 \\
\hline 88, & . & . & • & White pine, & & 6 & 7 & 2 & 35 & 58 & 23 \\
\hline 89 , & . & . & • & White pine, & & 4 & 4 & 3 & 10 & 15 & 5 \\
\hline 98, & . & . & & White pine, & . & 11 & 12 & 1 & 115 & 150 & 35 \\
\hline
\end{tabular}


Sample Plot No. 4 (Government Plot No. 7) - Concluded.

\begin{tabular}{|c|c|c|c|c|c|c|c|c|}
\hline \multirow[t]{2}{*}{ TreE } & \multirow[t]{2}{*}{ NUMBER. } & \multirow[t]{2}{*}{ Species. } & \multicolumn{2}{|c|}{$\begin{array}{c}\text { DIAMETER } \\
\text { BREAST HIGH } \\
\text { (INCHES). }\end{array}$} & \multirow[t]{2}{*}{ Class. } & \multicolumn{2}{|c|}{ BoArd Feet. } & \multirow{2}{*}{$\begin{array}{l}\text { Gain } \\
\text { in } \\
\text { Board } \\
\text { Feet. }\end{array}$} \\
\hline & & & 1905. & 1914. & & 1905. & 1914. & \\
\hline 99, & . & White pine, & 5 & 5 & 2 & 20 & 35 & 15 \\
\hline 101 , & . & White pine, & 6 & 7 & 2 & 35 & 58 & 23 \\
\hline 102 , & . & White pine, & 8 & 9 & 1 & 65 & 93 & 28 \\
\hline 105, & . & White pine, & 5 & 5 & 3 & 20 & 35 & 15 \\
\hline 106 , & . & White pine, & 6 & 6 & 3 & 35 & 48 & 13 \\
\hline 107, & . & White pine, & 6 & 6 & 3 & 35 & 48 & 13 \\
\hline 108, & . & White pine, & 6 & 6 & 3 & 35 & 48 & 13 \\
\hline 109, & . & White pine, & 6 & 6 & 3 & 35 & 48 & 13 \\
\hline 110, & . & White pine, & 5 & 5 & 3 & 20 & 35 & 15 \\
\hline 111 , & . & White pine, & 8 & 8 & 2 & 65 & 75 & 10 \\
\hline 112 , & . & White pine, & 11 & 12 & 1 & 115 & 150 & 35 \\
\hline 113 & . & White pine, & 8 & 8 & 1 & 65 & 75 & 10 \\
\hline Tota & & . & - & - & - & 3,345 & 4,835 & 1,490 \\
\hline
\end{tabular}

Average height, 1905, 60 feet. Average height, 1914, 65 feet.

- Summary of Data.

Total board feet to acre, 19C5, . . . . . . 26,760

Total board feet to acre, 1914, . . . . . . 38,680

Total gain in board feet per acre, . . . . $\quad$. 11,920

Current annual gain in board feet per acre, . . . 1,324

South Lancaster, Mass. - Owner, Mrs. Abbie F. Kilburn.

History. - This plantation joins that of Mr. Harold Parker, only a stone wall separating the two lots. It was made by Mr. Jonas Goss, and was planted in two sections, aged sixty years and forty-one years, respectively. The spacing was 6 by 8 feet. Since that time trees of various ages have been added around the edges. A survey was therefore necessary to show the areas of the original lots of even aged trees, which are: for the sixty-year old lot, 2.11 acres, and for the forty-one year old lot, 2.02 acres. If all trees are included, the area is 7 acres, and the figures given under "Treatment" were worked out on that basis by Mr. W. G. Kilburn.

Treatment. - In 1908 a thinning was made under the direc- 


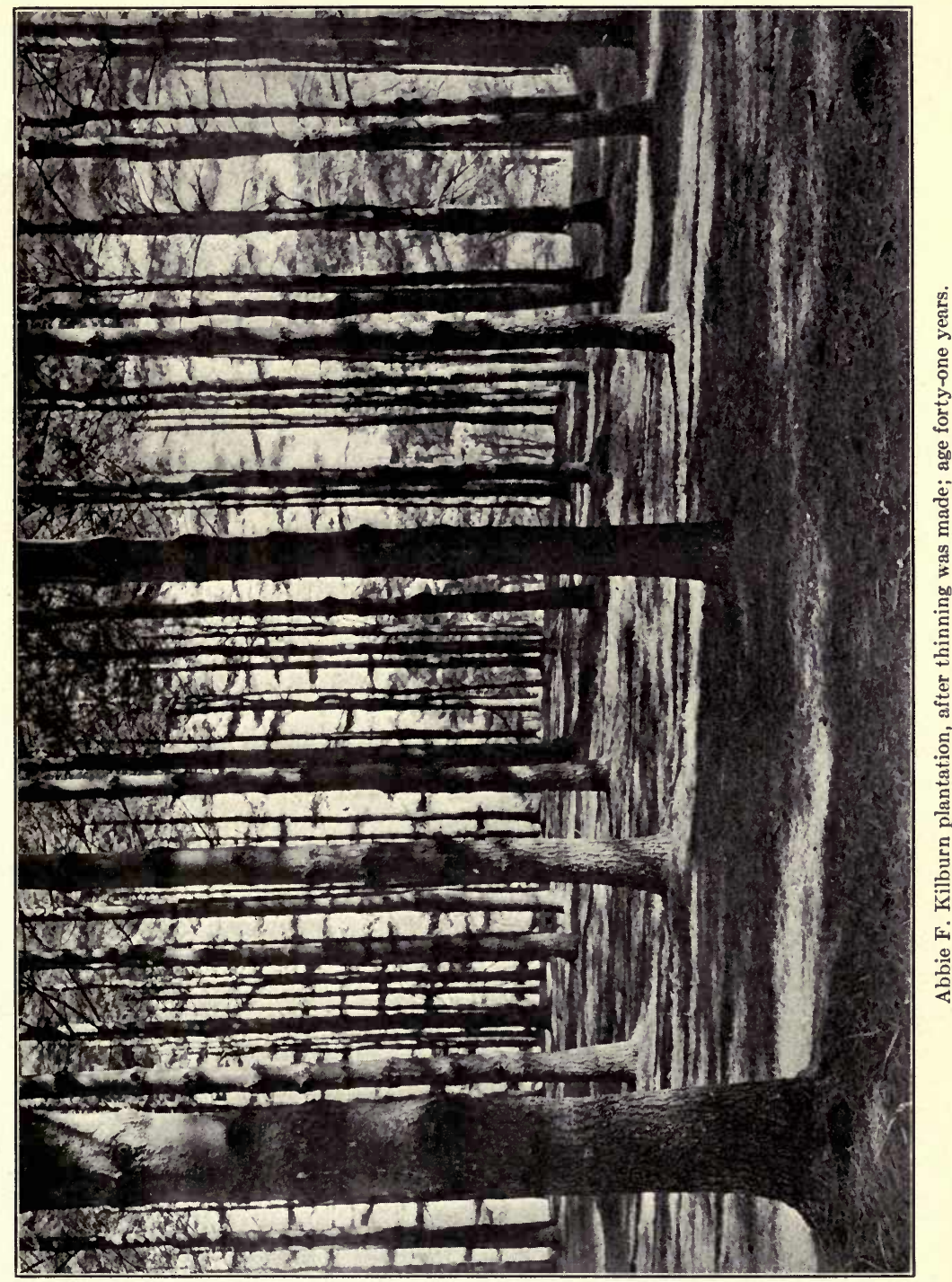



tion of this office, and about one-third of the trees removed. The following is a record of the lumber taken out:-

Box boards,

Wood,

Cost of brush burning,

Stumpage price received for lumber,

Stumpage price received for wood,

Profit per acre,

14,000 feet.

40 cords.

$\$ 35$

Net profit,

$\$ 8$ per M.

$\$ 1$ per cord.

$\$ 20$

$\$ 150$

While this work was going on the small dead branches so persistent on white pine were removed from the trunks of the trees in order to improve the general appearance of the grove. The thinning was not made so much with the idea of an immediate profit, as to increase the value of the plantation. While six years is not sufficient time to judge accurately as to the increase in growth due to thinning, the trees have every appearance of being stimulated by the added light received. This is shown by the healthy bark, green tops and absence of any dead or dying trees.

Section 1.

Area, 2.11 acres; age, sixty years.

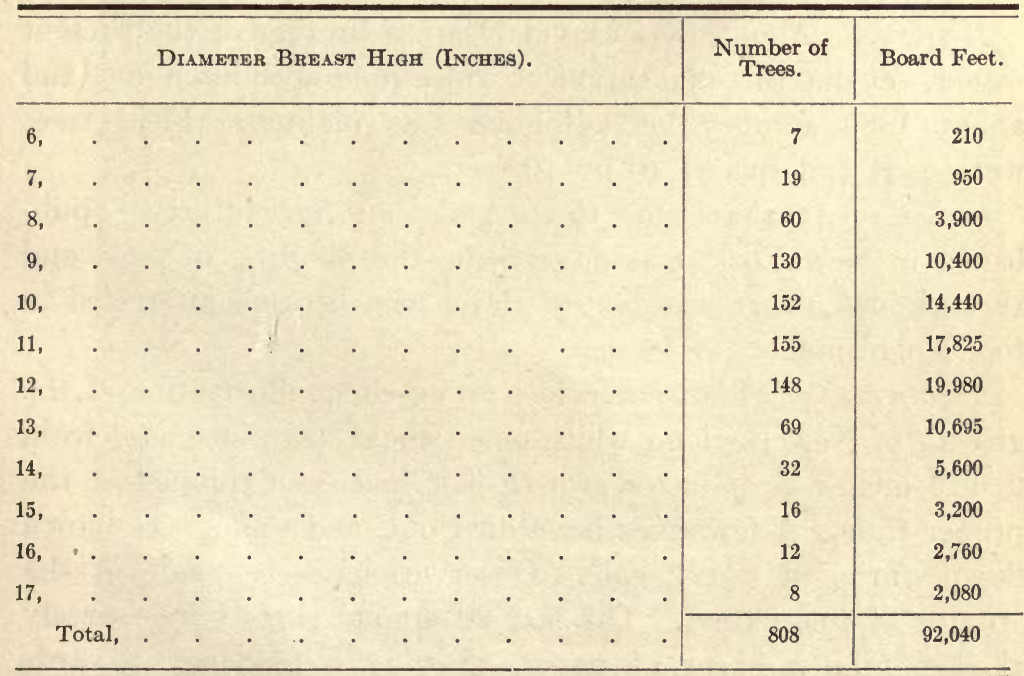

Total number of trees to acre, 383 .

Total board feet to acre, 43,620 .

Average height, 60 feet. 
Section 2.

Area, 2.02 acres; age, forty-one years.

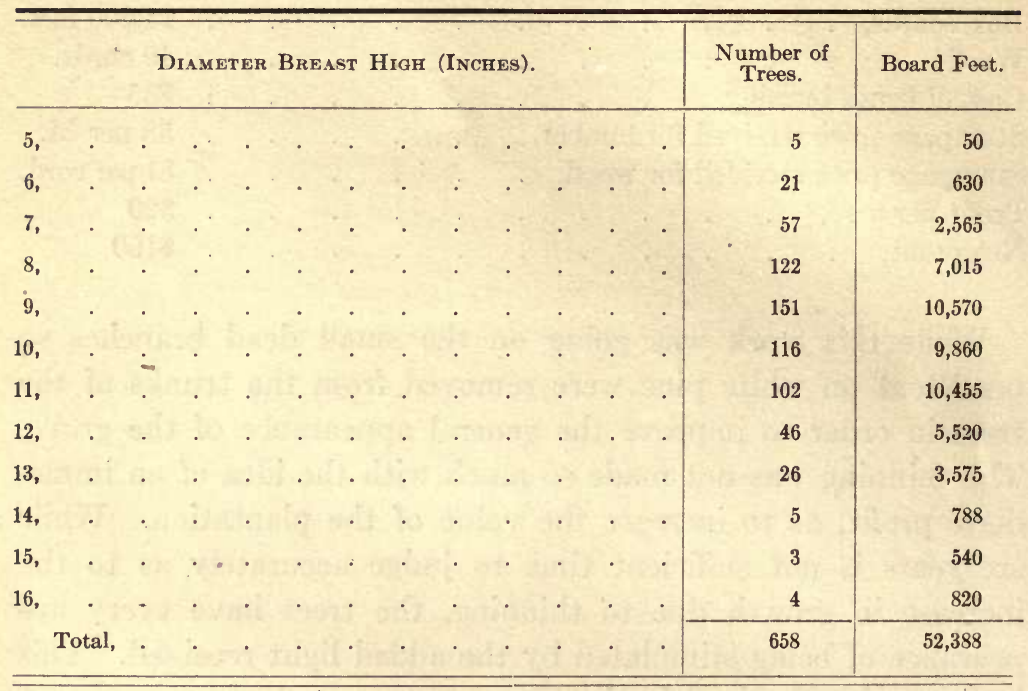

Total number of trees to acre, 325 .

Total board feet to acre, 25,934 .

Average height, 55 feet.

East Taunton, Mass. - Owner, Miss Margaret Dean.

History. - About 1854 David Dean, a brother of the present owner, set out this plantation of white pine near his home, and about 1880 another lot adjoining was planted. Field trees were used and spaced 10 by 10 feet.

Object. - At that time the "American Agriculturist," published in New York, was advertising the planting of pine, and the original owner was one of those who became interested in forest planting.

Treatment. - This tract, while an excellent illustration of the growth of New England white pine, would have increased from 2 to 3 inches in diameter growth if it had been thinned at the proper time. A few trees have died out, and some were blown down during a heavy gale. Observations were made of the stumps of these trees. The last 20 annual rings were scarcely thicker than a sheet of paper, showing a tendency towards stagnation. For the first twenty years the rings show a growth, in some cases, better than normal. 


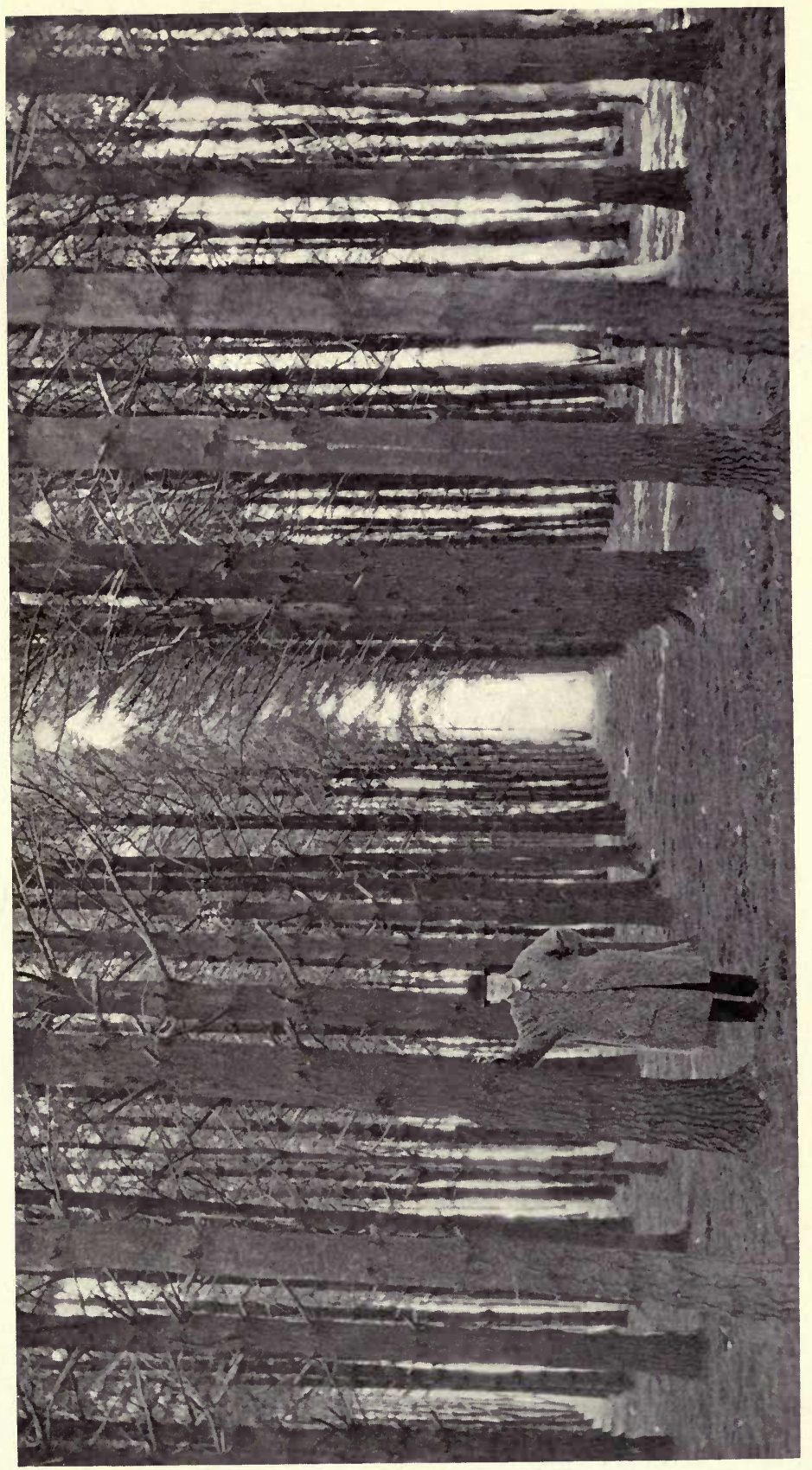

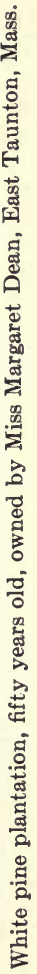



The measurements were made early in the year (1914), and the total results placed upon the records in the State Forester's office. The inch classes are therefore omitted.

Section 1, Sixty-one Year Old Plantation.

Area, 11/2 acres.

Total number of trees, 400 .

Average height, 60 feet.

Board feet to acre, 41,000 .

Section 2. Thirty-eight Year Old Plantation.

Area, $1 \frac{1}{2}$ acres.

Total number of trees, 375 .

Average height, 50 feet.

Board feet to acre, 29,000.

Georgetown, Mass. - Owner, H.E. Guptill.

History. - Although this timber was measured in 1912, the totals are here given to show the results of the growth of white pine planted with Norway spruce. The plantation was fortyeight years old when measured, and was made with natural seedlings transplanted from the woods.

\section{White Pine and Norway Spruce.}

Area, 2.57 acres; age, forty-eight years.

Total board feet of spruce, 27,000 .

Total board feet of pine, 55,000 .

Total stand per acre, 33,000 board feet.

\section{Conclusion.}

The total results of the investigation, in terms of board measure for pine, are as follows. The two sample plots of tamarack and the forty-two roadside trees at Sharon, Mass., are not included.

Plantations 30 to 40 years old,

Board Feet per Acre.

Plantations 40 to 50 years old, . . . . . . . . 32,726

Plantations 50 to 60 years old, . . . . . . . . . 41,186 
These results compare favorably with measurements made by this office of healthy stands of native Massachusetts white pine. No attempt has been made to show that planted pine, untreated, would produce more. On only two of the plantations was anything like a systematic thinning made (pages 18 and 31 ), and in no case were trees removed at an age when an added amount of light would have given the remaining trees the maximum amount of growth. The present method of planting calls for a 6 by 6 foot spacing, but with the idea that thinning is to be done as the trees develop to prevent overcrowding, to realize something in the way of an income on the plantation, and to develop the best diameters and heights possible for the final stand.

In conclusion the reader is referred to the yield tables on pages 37 and 38, representing the financial rotations of white pine plantations, the one under the present general system of taxing forest land, and the other under the new forest taxation law enacted in 1914. These tables are compiled by H. O. Cook, supplementary to the financial rotations in his bulletin on "Forest Mensuration of the White Pine." The volume tables used in this investigation were taken from the same bulletin.

When we speak of the yield of a pine plantation we think principally of the amount of lumber which can be cut from the land. Now, while this is important, the real determining factor as to whether a plantation is a success or not is the financial profit that such a plantation will bring. As with any investment, it is not the amount of business done, but the profits made which in the last analysis determine failure or success.

Timber crops are peculiar in that they cannot be harvested except after a period of years, so that it is not alone necessary to deduct actual expenses from gross returns in order to determine net yield, but these expenses must be carried from the date of their incurrence at some determined rate. As most savings banks pay 4 per cent., we must use a rate better than that, and have chosen 5 per cent. The net yield is in this case, therefore, a speculative profit over and above 5 per cent.

The gross returns are in this case the stumpage value ob- 
tained from a yield table made by measuring sample plots in well-stocked natural stands in all parts of this State. Natural stands were taken because of the limited number of plantations that can be taken for this purpose. It will be seen on comparison, however, that the amounts given in the table are in substantial agreement with the average shown by the plantations described in this bulletin. The stumpage rates chosen run from $\$ 6$ to $\$ 10$, and are the rates of the present day, no allowance being made for the increase that future years will bring.

In compiling these tables certain assumptions were made, based on actual experience. The cost of the land is assumed to be the first expense, and is placed at $\$ 5$ per acre. The second premise is the cost of planting which is placed at $\$ 12$ per acre. These two expenses must be carried at compound interest from the beginning to the end of the rotation.

We have presented two tables, one of which shows the returns of a plantation taxed under the present general system, and the other for the same plantation registered under the new taxation law. We have assumed the tax rate to be $\$ 20$ per thousand. The land, of course, pays taxes from the beginning, but the timber not until the twenty-fifth year. In the case of classified land it pays no tax until it is cut, when it pays a product tax of 6 per cent. All of these expenses are carried at 5 per cent. compound interest to the end of the rotation, and we see that in the case of unclassified land the maximum yield comes at fifty years, when there is an excess profit of $\$ 60$. In the case of registered land the maximum yield comes at fifty years, and there is an excess profit of $\$ 140$, or two and a half times that of the unregistered. It should be said here, however, that in the case of the unclassified land we have assumed that the assessors taxed it at its full value, which is unusual in practice.

We would urge that every owner of a young artificial plantation should register his land, for it will be seen that while the owner of Plantation 1 invested in actual cash $\$ 83$ on his timber in fifty years, the owner of the registered Plantation 2 only risked $\$ 40$ in cash. On account of the time element and risk of a forest plantation the owner should take every opportunity 
of keeping down his money investment. At present prices on lumber, a pine plantation is a 6 or 7 per cent. investment in Massachusetts, but an increase in stumpage values of whatever per cent. will make a corresponding increase in the returns. In other words, the planter of the present day can assume that he is investing for a 10 or 12 per cent. return rather than a 6 or 7 per cent. return. 


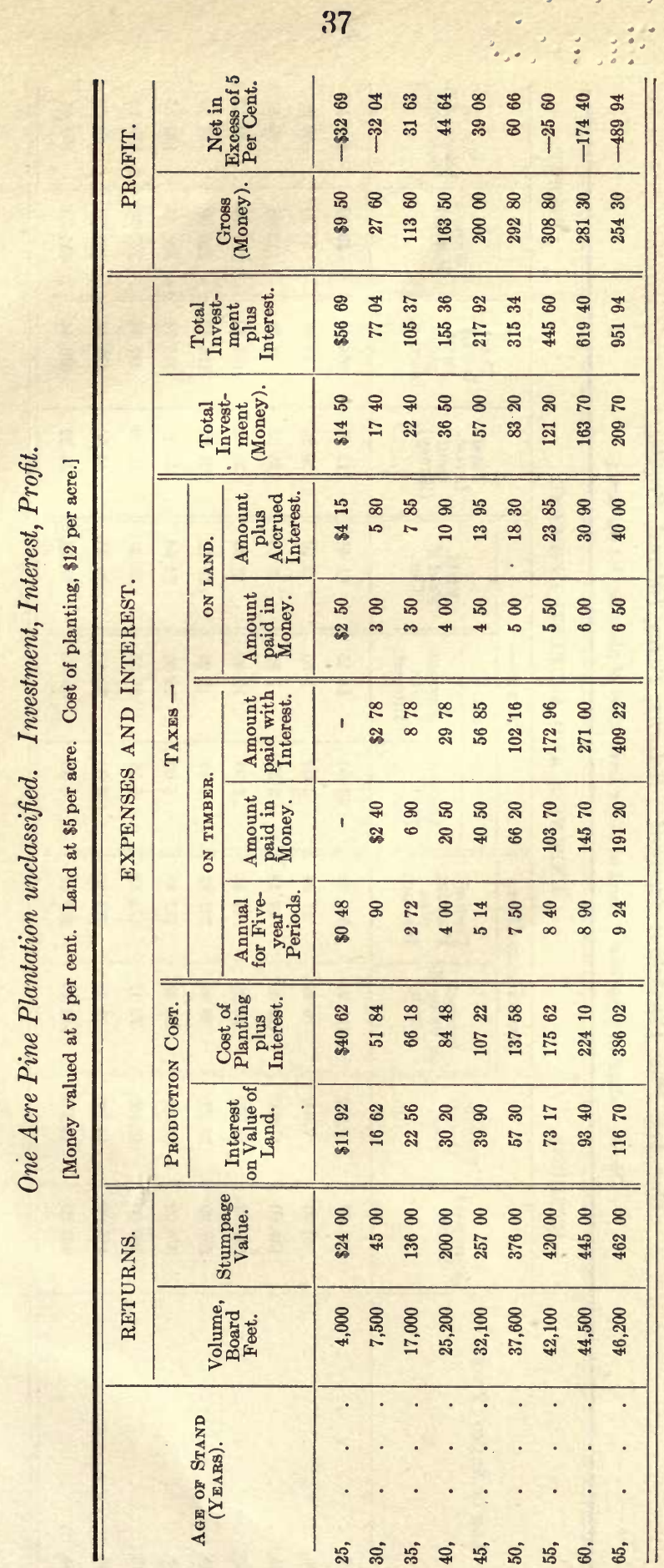




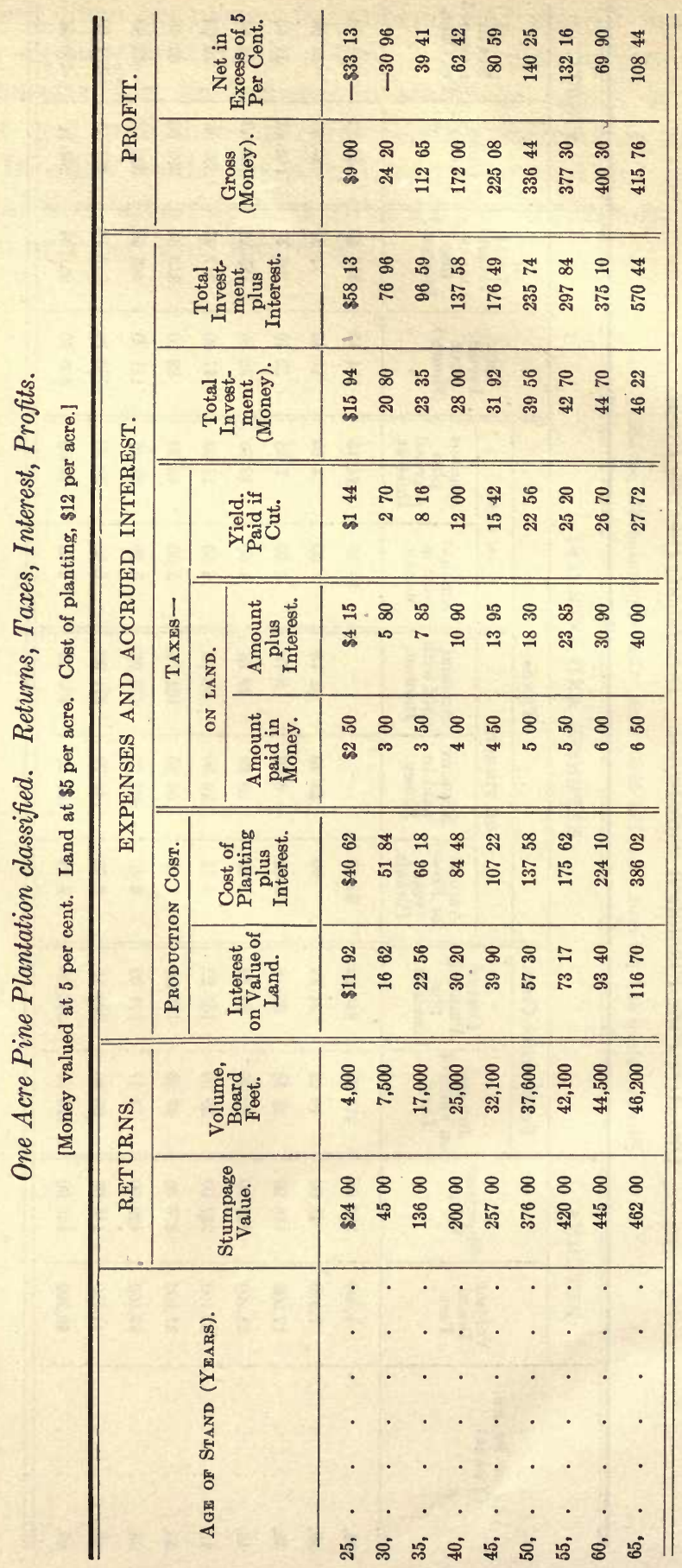











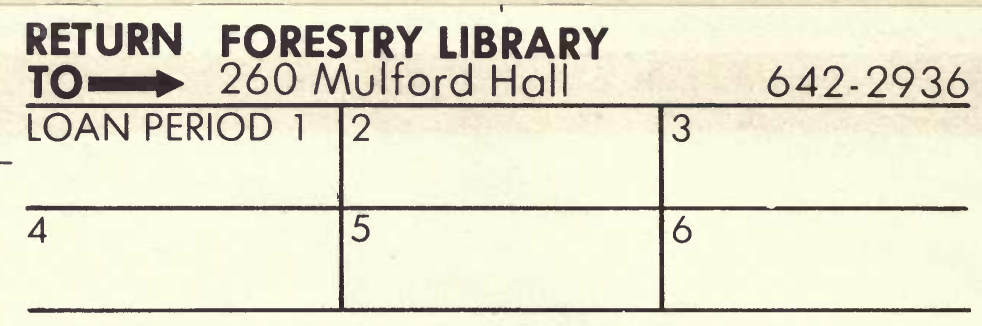

ALL BOOKS MAY BE RECALLED AFTER 7 DAYS

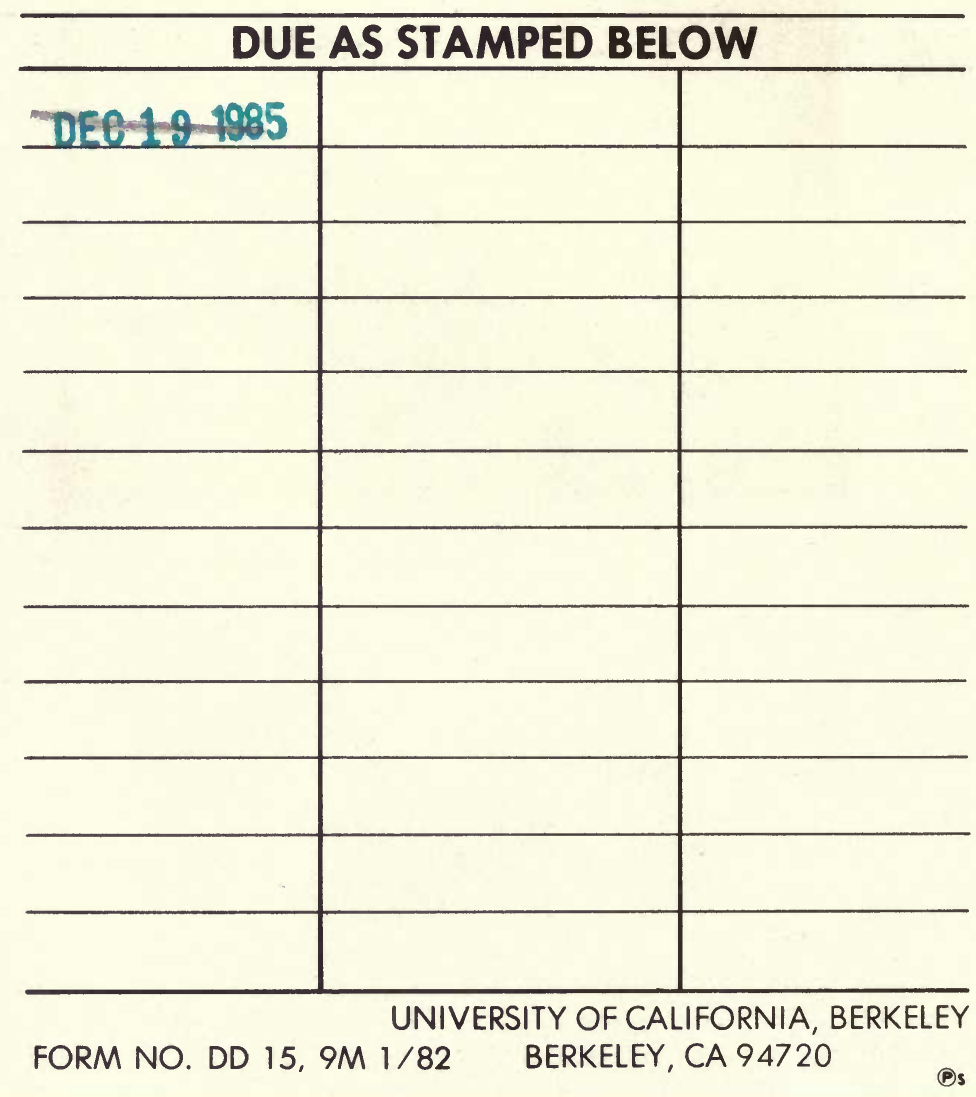



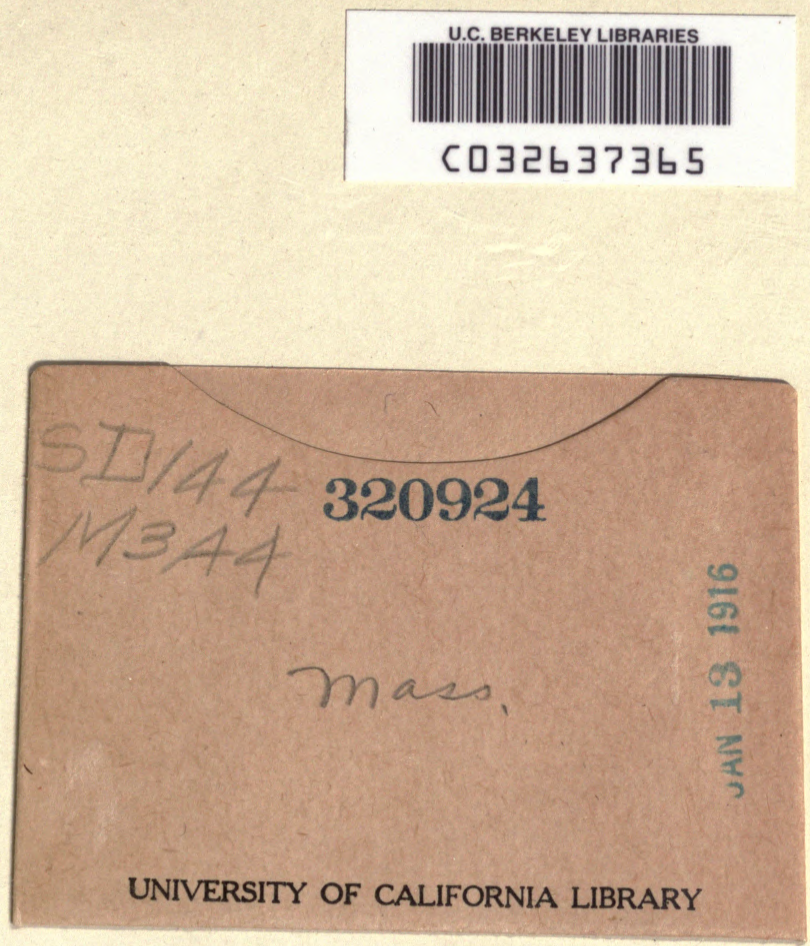
